no. SL-95-16

c. 2

\title{
Corps
}

eers

Waterways Experiment

Station

Supporting Studies for Explosives Safety Program

\section{Analysis of Heat-Activated Explosions in Storage of HD 1.2 Munitions}

\section{SSES Report No. 1}

\author{
by Benny L. Carnes, WES \\ John S. Furey, Computer Science Corporation \\ Morris P. Fields, Illinois Institute of Technology Research Institute
}

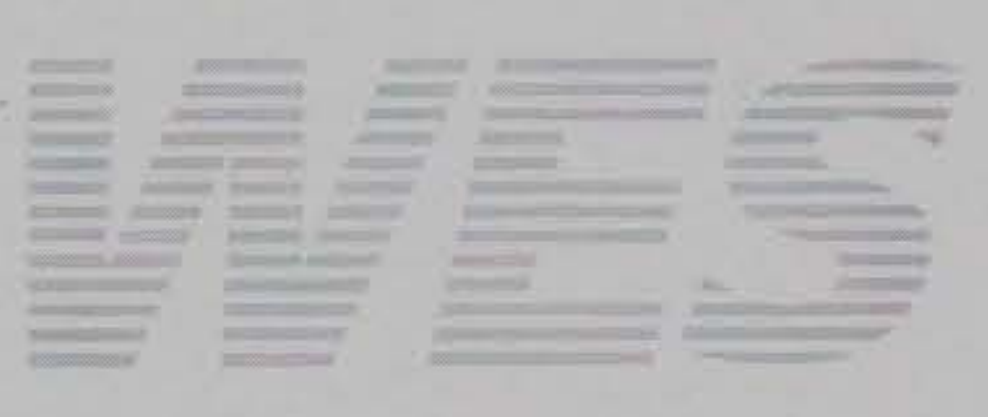

Approved For Public Release; Distribution Is Unlimited

Prepared for Office of the Under Secretary of Defense for Acquisition 
The contents of this report are not to be used for advertising, publication, or promotional purposes. Citation of trade names does not constitute an official endorsement or approval of the use of such commercial products.

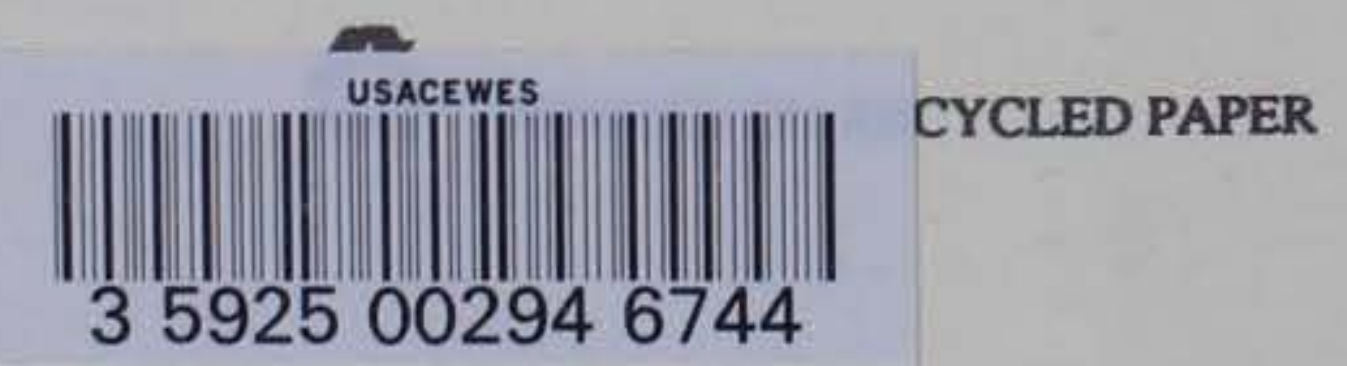




\section{Analysis of Heat-Activated Explosions in Storage of HD 1.2 Munitions}

\section{SSES Report No. 1}

by Benny L. Carnes

U.S. Army Corps of Engineers

Waterways Experiment Station

3909 Halls Ferry Road

Vicksburg, MS 39180-6199

John S. Furey

Computer Science Corporation

Vicksburg, MS 39180-5378

Morris P. Fields

Illinois Institute of Technology Research Institute

Dayton, $\mathrm{OH} \quad$ 45432-3036

Final report

Approved for public release; distribution is unlimited

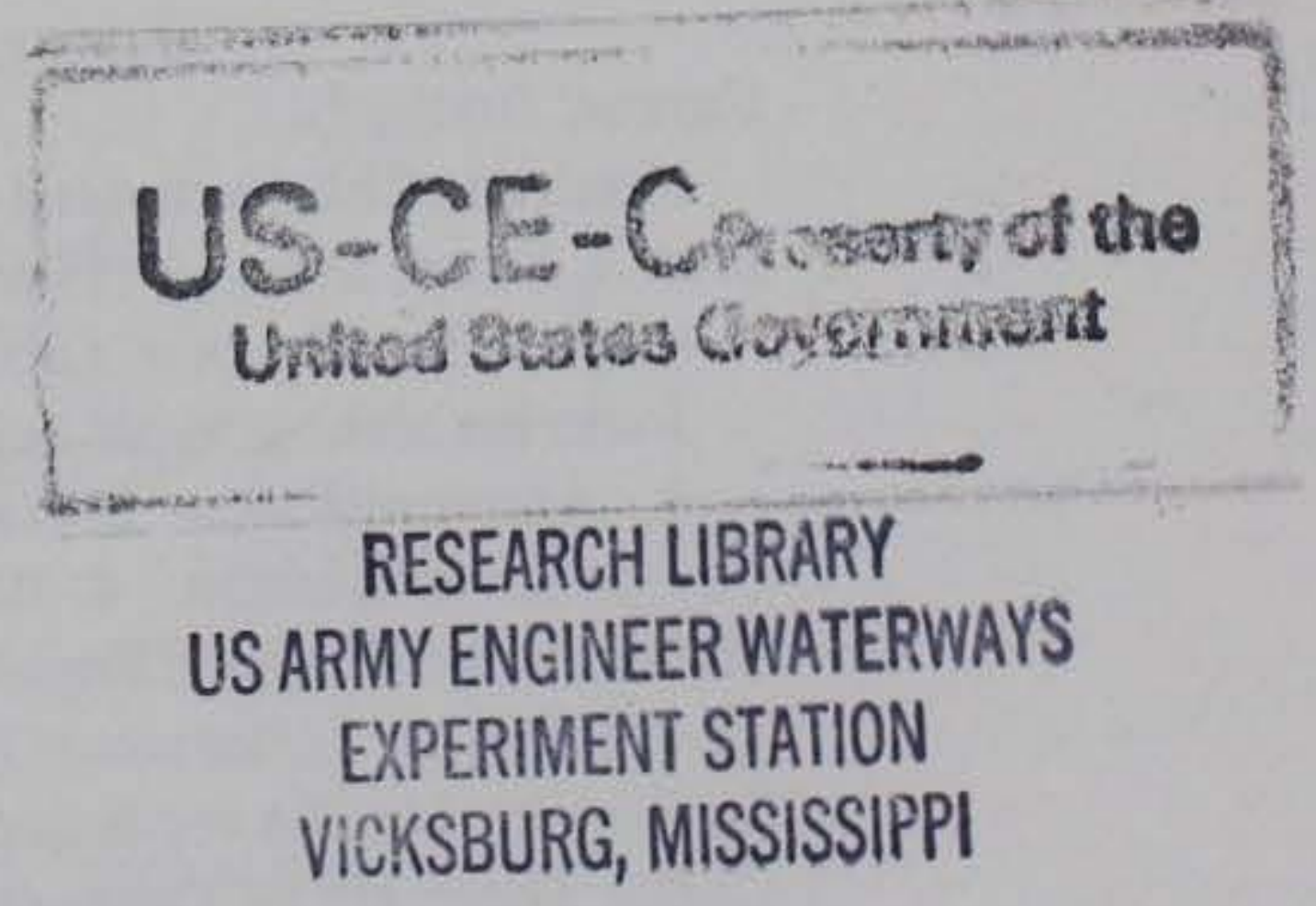

Prepared for Office of the Under Secretary of Defense for Acquisition Washington, DC 20315 


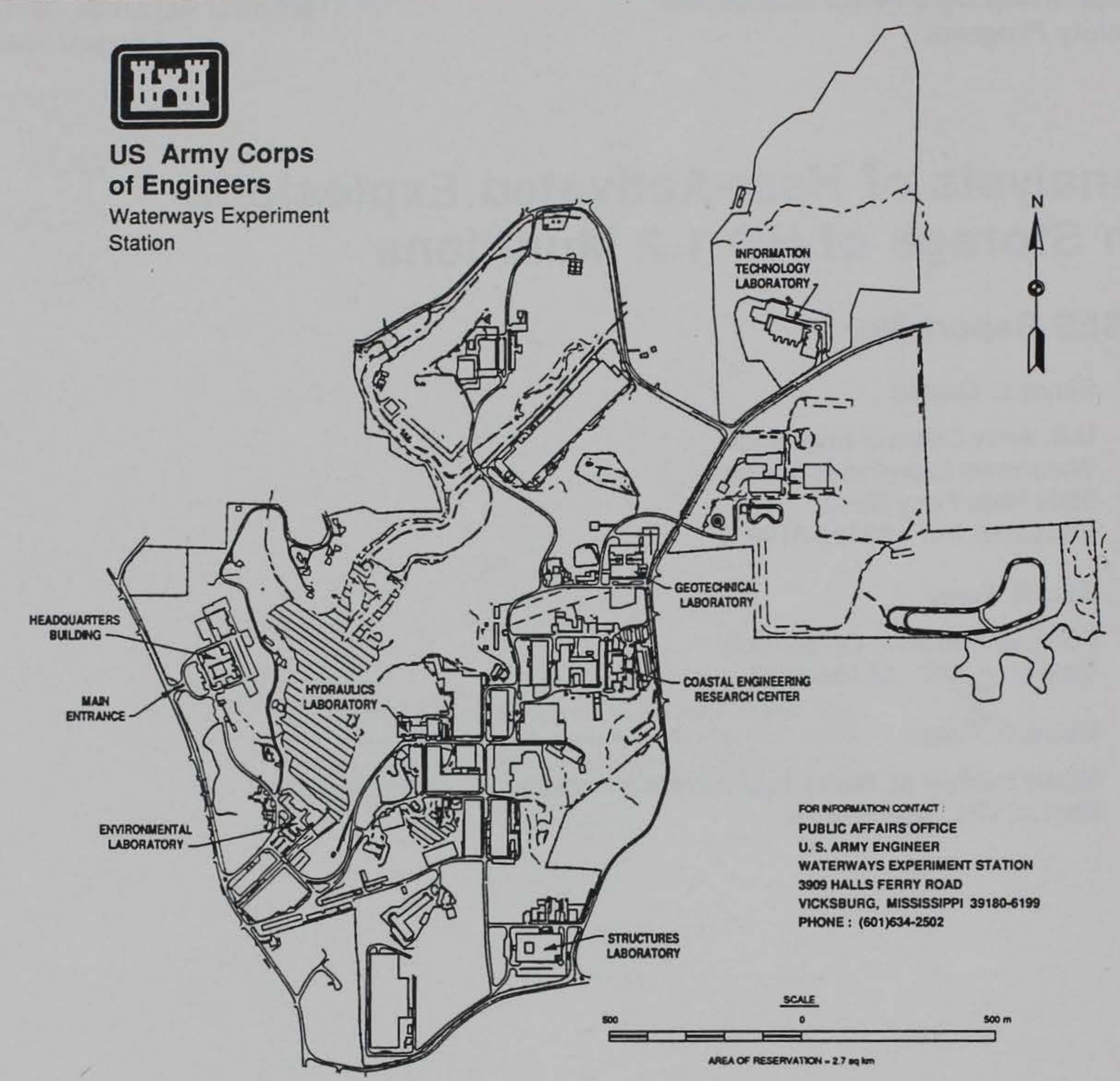

\section{Waterways Experiment Station Cataloging-in-Publication Data}

Carnes, Benny L.

Analysis of heat-activated explosions in storage of $\mathrm{HD} 1.2$ munitions / by Benny L. Carnes,

John S. Furey, Morris P. Fields ; prepared for the Under Secretary of Defense for Acquisition. 40 p. : ill. ; $28 \mathrm{~cm}$. - (SSES report ; no. 1)(Technical report ; SL-95-16)

Includes bibliographic references.

1. Ammunition -- Storage. 2. Explosives, Military -- Accidents. 3. Explosions -Computer simulation. 4. Blast effect. I. Furey, John S. II. Fields, Morris P. III. United States. Army. Corps of Engineers. IV. U.S. Army Engineer Waterways Experiment Station. V. Structures Laboratory (U.S. Army Engineer Waterways Experiment Station) VI. Supporting Studies for Explosives Safety Program (U.S.) VII. United States. Office of the Under Secretary of Defense for Acquisition (Research and Advanced Technology) VIII. Title. IX. Series: SSES report ; no. 1 X. Series: Technical report (U.S. Army Engineer Waterways Experiment Station); SL-95-16.

TA7 W34 no.SL-95-16 


\section{Contents}

Preface $\ldots \ldots \ldots \ldots \ldots \ldots \ldots \ldots \ldots \ldots \ldots$

1-Introduction $\ldots \ldots \ldots \ldots \ldots \ldots \ldots \ldots \ldots \ldots \ldots$

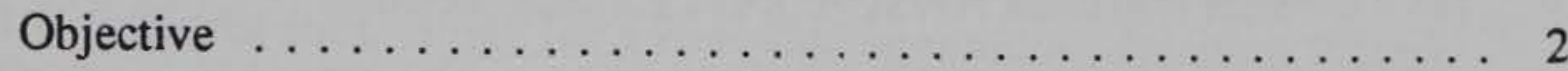

2-Theoretical Considerations $\ldots \ldots \ldots \ldots \ldots \ldots \ldots \ldots$

Description of Simulations $\ldots \ldots \ldots \ldots \ldots \ldots \ldots \ldots$

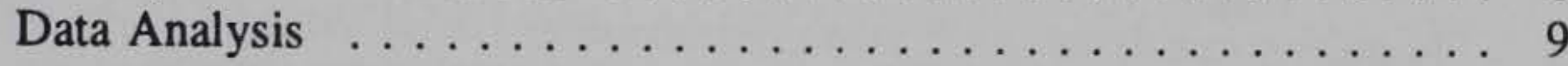

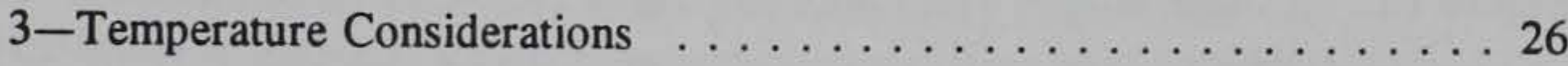

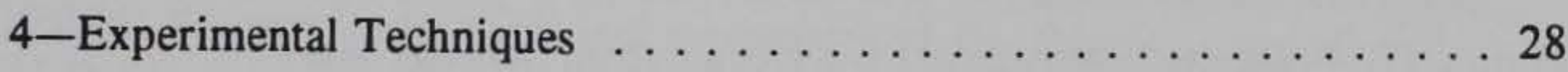

NSWC and USATCES Tests $\ldots \ldots \ldots \ldots \ldots \ldots \ldots \ldots$

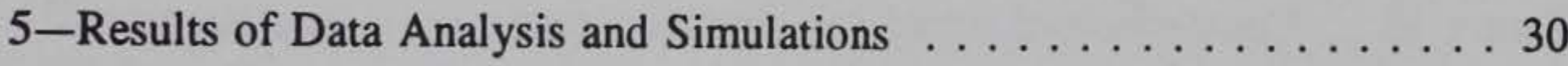

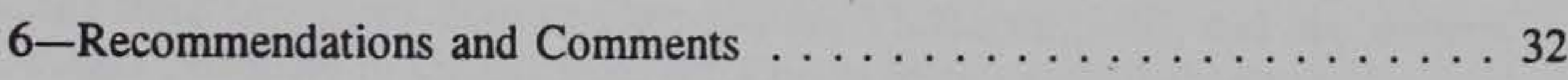

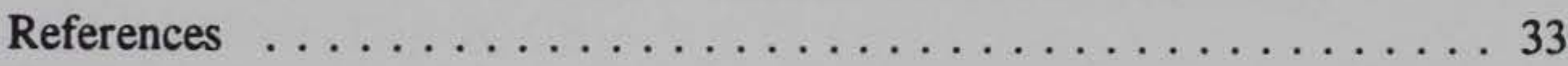

\section{List of Figures}

Figure 1. Explosion rates in the semi-microscopic theory for several values of the heating parameter $h \ldots \ldots \ldots \ldots$

Figure 2. The Gumbel Distribution $\exp (-\exp (-\mathrm{x}))$ for a generic quantile $x \ldots \ldots \ldots \ldots \ldots \ldots \ldots \ldots$

Figure 3. Histogram of explosion times from a Monte Carlo simulation $\ldots \ldots \ldots \ldots \ldots \ldots \ldots \ldots$

Figure 4. A portion of waveform from WES Test \#12 plotted at two scales $\ldots \ldots \ldots \ldots \ldots \ldots \ldots \ldots \ldots \ldots \ldots \ldots \ldots \ldots \ldots$

Figure 5. The two definitions of rate for WES Test \#12, with a time

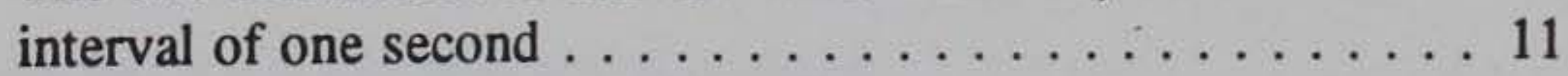

Figure 6. The two definitions of rate for WES Test \#12, with a time interval of ten seconds $\ldots \ldots \ldots \ldots \ldots \ldots \ldots \ldots \ldots$ 
Figure 7. Maximum number of events for Test $\# 12$ versus time interval . . . . . . . . . . . . . . 13

Figure 8. Explosion rates for WES Test \#13 (top) and NSWC Test \#6 (bottom) . . . . . . . . . . . . . . . 15

Figure 9. WES Test \#11 (top) and Test \#12 (bottom) $\ldots \ldots \ldots 16$

Figure 10. Two simulated tests of 70 munitions in identical fire

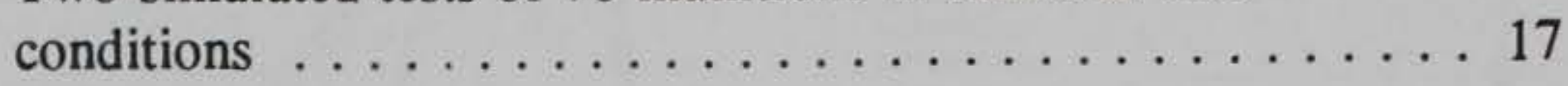

Figure 11. Normalized rate for the total of WES tests $\ldots \ldots \ldots \ldots 18$

Figure 12. Normalized rate for the total of NSWC and USATCES tests . . . . . . . . . . . . . . . . . 19

Figure 13. Hazard rates shown with normalized rates for WES tests (top) and NWSC-USATCES tests (bottom) . . . . . 21

Figure 14. Gaussian fit to WES tests produced a poor result $\ldots \ldots \ldots 22$

Figure 15. Gumbel fit to NSWC-USATCES tests produced a good

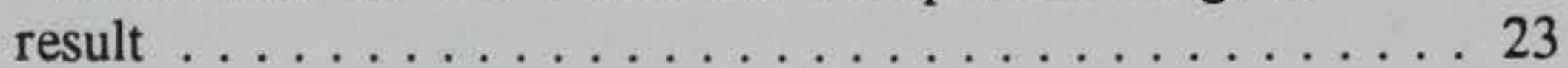

Figure 16. Comparison of WES tests and scaled NSWC-USATCES

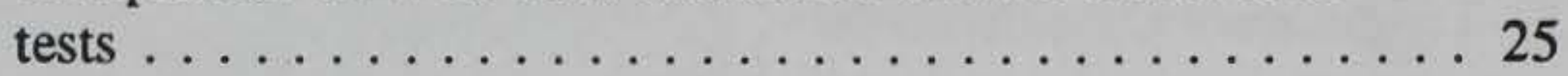

Figure 17. Selected temperature waveforms from Swisdak and Rye (1994) . . . . . . . . . . . . . . . 27

Figure 18. Comparison of actual and simulated tests $\ldots \ldots \ldots \ldots 31$ 


\section{Preface}

This study was conducted by the U.S. Army Engineer Waterways Experiment Station (WES), under a program entitled "Supporting Studies for Explosives Safety (SSES)." The SSES program was a special adjunct of the Joint U.S./Korea R\&D Study for a New Underground Ammunition Storage Technologies (UAST). Funding for the SSES program was provided by the Office of the Under Secretary of Defense for Acquisition.

The study reported here was conducted under the general supervision of Mr. Bryant Mather, Director, Structures Laboratory (SL), WES, and Dr. Jimmy P. Balsara, Chief, Geomechanics and Explosion Effects Division (GEED), SL, and under the direct supervision of Dr. Benny L. Carnes, GEED.

This report was prepared by Dr. Carnes, Mr. John S. Furey (assigned to GEED from Computer Science Corporation, Vicksburg, MS), and Mr. Morris P. Fields (assigned to GEED from the Illinois Institute of Technology Research Institute, Dayton, $\mathrm{OH})$.

The Technical Manager of the UAST Program was Mr. L. K. Davis, GEED. The UAST Program Manager was Mr. Gary L. Abrisz, U.S. Army Technical Center for Explosive Safety (USATCES), Savanna, IL. The SSES program was performed under the direct supervision of Mr. Davis. Messrs. Kenyon Williams and Richard Cashin, USATCES, were technical monitors of the SSES program.

The assistance provided by Mr. John C. Morgan, formerly assigned to GEED from the Illinois Institute of Technology Research Institute, in the conduct of this study is gratefully acknowledged.

At the time of publication of this report, the Director of WES was Dr. Robert W. Whalin. The Commander was COL Bruce Howard, EN.

The contents of this report are not to be used for advertising, publication, or promotional purposes. Citation of trade names does not constitute an official endorsement or approval for the use of such commercial products. 


\section{Introduction}

There are many factors to consider in designing safe ammunition storage areas and in developing optimal storage configurations. The most important safety factor is the Quantity-Distance, or "QD", which defines the acceptable hazard range from an accidental explosion of the stored munitions. The QD is based on a Maximum Credible Event (MCE), which is the greatest quantity of explosive material in the storage area that could be expected to detonate as (essentially) a single event. Ammunition and explosives that are likely to mass detonate are designated by the DOD Ammunition and Explosive Safety Standards (DOD 6055.9-STD, 1992) as Hazard Division (HD) 1.1 materials. Other types of ammunition and explosives that are not expected to mass detonate, but may explode individually under conditions such as a fire in the storage area, are designated as HD 1.2 materials.

Ammunition may be stored in an open area, or inside specially-designed storage structures. Above-ground structures containing large amounts of HD 1.1 material normally are not intended to contain the effects of an accidental explosion; rather, they are designed to protect the material inside from being sympathetically detonated by the effects of another nearby explosion. Consequently, relatively large land areas may be encumbered as safety buffer zones to meet the QD requirements for storage of HD 1.1 material. For storage of HD 1.2 material, however, the structure may often be designed to contain the blast and fragment hazards from internal explosions. Thus, the structure itself can reduce the external QD for an internal explosion to essentially zero.

For HD 1.2 ammunition stored in the open or in buildings of light construction, a primary external hazard is fragmentation produced by rounds that individually detonate as they "cook-off" in a fire. The U.S. and the United Kingdon (U.K.) are currently jointly funding a test program conducted by the Naval Surface Warfare Center (NSWC), White Oak Detachment, to define the fragment hazard range for several types of HD 1.2 munitions, including $105-\mathrm{mm}$ artillery and $81-\mathrm{mm}$ mortar rounds. Palletized stacks of boxes containing the munitions are burned in bonfires in an open area (Swisdak and Rye, 1994) to record the number of cook-off detonations that occur. Similar bonfire tests have been conducted by the U.S. Army Technical Center for Explosive Safety (USATCES) on 120-mm rounds (Williams, 1994). 
While these tests provide very valuable data, a number of questions remain concerning other realistic storage situations. The bonfire tests results support the current idea that heat-activated explosions of HD 1.2 munitions are individual detonations, but for large quantities of munitions the time intervals between individual detonations could become very short, so that multiple detonations may occur within a critical time interval. This effect could produce a much larger MCE than the detonation of an individual munition.

If too many detonations occur within a sufficiently short time interval inside a storage structure, the structure could be blown apart by the pressure from the combination of multiple blast waves, and thus lose its fragmentcontaining capability for subsequent detonations. Since some storage structures have limited venting areas, the individual explosions can occur so rapidly that gas pressures build up faster than they can be vented, which could also blow apart the structure. Furthermore, it is conceivable that, if a significant number of rounds explode individually in a rapid sequence of heatactivated explosions inside a robust storage structure, the change of storage conditions resulting from the increase in heat, pressure, and shock-sensitizing of the remaining rounds could cause a mass detonation.

In open storage bonfire tests, many rounds do not detonate because they are thrown from the fire by the explosions of other rounds. If confined in a storage structure, however, the rounds cannot be scattered past the structure walls to remove them from further heating. Additionally, the heat from the fire and previous explosions does not dissipate as quickly inside a structure as it does in the open. Consequently, the percentage of rounds which undergo heat-activated explosions in a fire inside a structure may be much greater than is indicated by open bonfire tests. To address these concerns, and to provide a larger data base of heat-activated explosions for greater statistical reliability, a number of experimental tests using small-arms ammunition were conducted at the U.S. Army Engineer Waterways Experiment Station (WES). These WES tests are described in detail later in this report.

The scenario to be considered in this study was that of a storage facility containing a specific quantity of HD 1.2 ammunition. The "worst case" situation assumes that the whole storage area catches fire and the heat level eventually initiates explosions throughout the entire munition storage. It is assumed that only the buildup of heat initiates the explosions, and explosions induced by fragment impact or airblast are not considered.

\section{Objective}

The objectives of this study were to (a) determine if cook-offs of HD 1.2 munitions displayed a characteristic time history pattern of detonations; (b) define the time history of explosions in the worst case scenario and evaluate the explosion statistics; and (c) determine the probability that a critical number of rounds may explode within a given time interval. 


\section{Theoretical Considerations}

Heat-activated explosions of HD 1.2 munitions occur when the most heat-sensitive part of the explosive charge (or the primary initiating train) is exposed to a sufficient temperature level for a sufficient length of time. The rate of occurrence of such explosions should be proportional to the number of munitions involved and to the probability of any one munition exploding. For a uniform chemical composition and temperature in a microscopic unit volume of explosive, the probability of initiating a heat-activated reaction is given by the Arrhenius theory (Holtzclaw, et. al., 1984). A semi-microscopic adaptation of the Arrhenius theory has the expression

$$
d N=-N * d t * R * \exp (-A / T)
$$

for the change in the number $N$ of undetonated munitions in the time interval $d t$, where $T$ is temperature, $A$ is the Arrhenius activation parameter, and $R$ is the reaction frequency factor. The rate of occurrence of explosions is then given by

$$
\text { rate }=-d N / d t
$$

For a constant uniform temperature $T_{0}$ and an original quantity $N_{0}$, the above expression for the number of undetonated munitions remaining integrates to

$$
N=N_{0} * \exp \left(-R * t * \exp \left(-A / T_{0}\right)\right)
$$

which is an exponential decrease with time. The maximum rate of explosions is

$$
\text { rate }_{\max }=N_{0} * R * \exp \left(-A / T_{0}\right)
$$


which occurs at the beginning, since the number of undetonated munitions is largest at that time. This exponential behavior is not observed for HD 1.2 munitions in fires, however, because during the course of a fire the temperature rises, causing the rate of heat-activated explosions to increase to a maximum and then decrease. For a given time-varying temperature, it is possible to integrate numerically to find the rate. In the general case of increasing temperature, the maximum rate occurs when

$$
d T=t^{2} * d t * R \exp (-A / T) / A
$$

The rate is not a simple function of the heating parameters, however, even if the temperature is a simple function of time. If the temperature increases linearly with time with a constant heating parameter $h$,

$$
T(t)=T_{0}+h * t
$$

Introducing $x(t)=-A / T(t)$ then leads to

$$
\text { rate } \left.\left.=N_{0} * R * \exp (x) t\right)\right) * \exp \left(-R * A / h \int \exp (x) d x / x^{2}\right)
$$

Figure 1 shows this rate plotted versus time for a wide range of heating parameter values, with the quantities expressed in dimensionless form; i.e., rate is divided by $N_{0} * R$, time is multiplied by $R$, and the heating parameter is divided by $R^{*} A$. It can be seen from Figure 1 that smaller values of the dimensionless heating parameter lead to smaller, more normally distributed explosion rates for the semi-microscopic theory.

For the carefully arranged compounds used in explosives, expressing the rate of explosions in terms of the semi-microscopic parameters requires that both the activation parameter and the frequency factor depend on the temperature. The relevant temperature in a storage situation will vary from munition to munition, because a fire will not heat the entire storage area uniformly. Hence, even a semi-microscopic theory must include the macroscopic statistics of the temperature distribution in order to predict the detonation rates that have been observed in experiments. The conditions under which a semi-microscopic theory is valid require a very small value of $d t$ and a very large $N$. Such a theory is difficult to simulate on a computer.

As an alternative, a macroscopic theory of heat-activated explosions recognizes the existence of a critical temperature at which a munition will explode. This critical temperature depends on the chemical composition of the explosive and, to some extent, the details of the manufacturing process, 

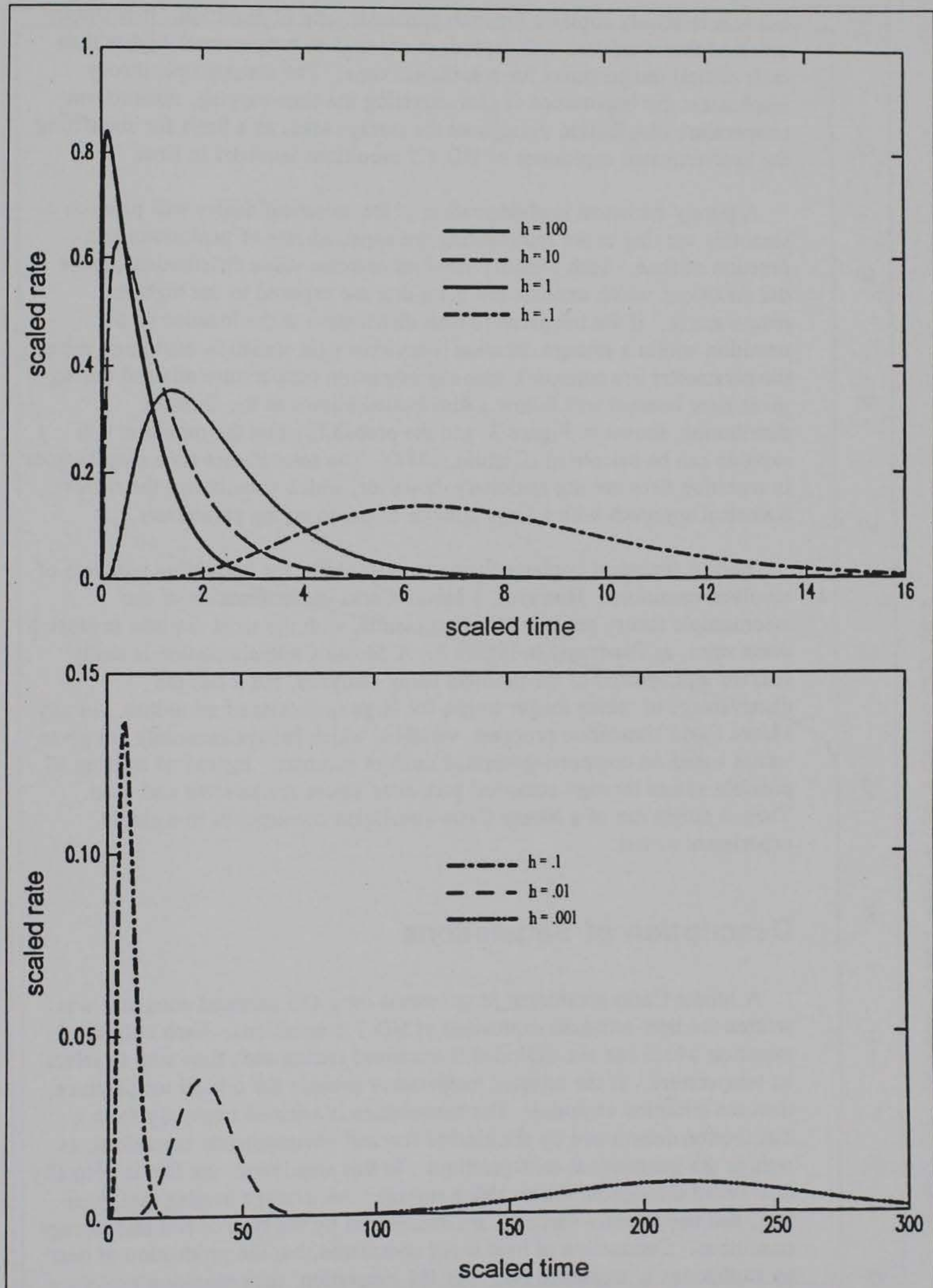

Figure 1. Explosion rates in the semi-microscopic theory for several values of the heating parameter $h$ 
but it is relatively constant for each particular type of munition. It is simply assumed that munitions will explode if exposed to temperatures higher than their critical temperatures for a sufficient time. The macroscopic theory emphasizes the importance of characterizing the time-varying, nonuniform temperature distribution throughout the storage area, as a basis for simulating the heat-activated explosions of HD 1.2 munitions involved in fires.

A purely statistical implementation of the empirical theory will produce a smoothly varying curve representing the expected rate of explosions as a function of time. Such a theory involves extreme value distributions, since the munitions which explode are those that are exposed to the highest temperatures. If the temperature-time distribution at the location of a munition within a storage situation is stationary (in statistics, stationary means the parameters are constant), then the maximum temperature attained during a given time interval will follow a distribution known as the Gumbel distribution, shown in Figure 2, and the probability that the munition will explode can be calculated (Castillo, 1988). The temperature-time distributions in munition fires are not stationary, however, which complicates the direct statistical approach with a large number of time-varying parameters.

Such a statistical implementation is most useful for very large numbers of involved munitions. However, a Monte Carlo implementation of the macroscopic theory produces the best results, with the most realistic explosion event rates, as illustrated in Figure 3. A Monte Carlo simulation is easily adapted and updated to the problem being analyzed, but it has the disadvantage of taking longer to run for large quantities of munitions. In any Monte Carlo simulation program, variables which behave randomly are given values based on computer-generated random numbers. Instead of treating all possible values through statistics, particular values are selected and used. Thus, a single run of a Monte Carlo simulation corresponds to a single experiment or test.

\section{Description of Simulations}

A Monte Carlo simulation programmed on a 486 personal computer was written for heat-activated explosions of HD 1.2 munitions. Each individual munition which has not exploded is examined during each time step to select its temperature. If the selected temperature exceeds the critical temperature, then the munition explodes. The temperature is selected randomly from a distribution determined by the kind of fire and environmental conditions, as well as the geometrical configurations. In this simulation, the fire is normally distributed throughout the munition storage. An average heating rate, heat loss, and temperature variation are determined by the type of fire and storage conditions. Conduction of heat is not considered, but the production of heat by explosions is accounted for. For the simulation, each munition explosion is assumed to produce a uniform distribution of fragments. The fragment effects are assumed to accumulate as each individual munition explodes. 


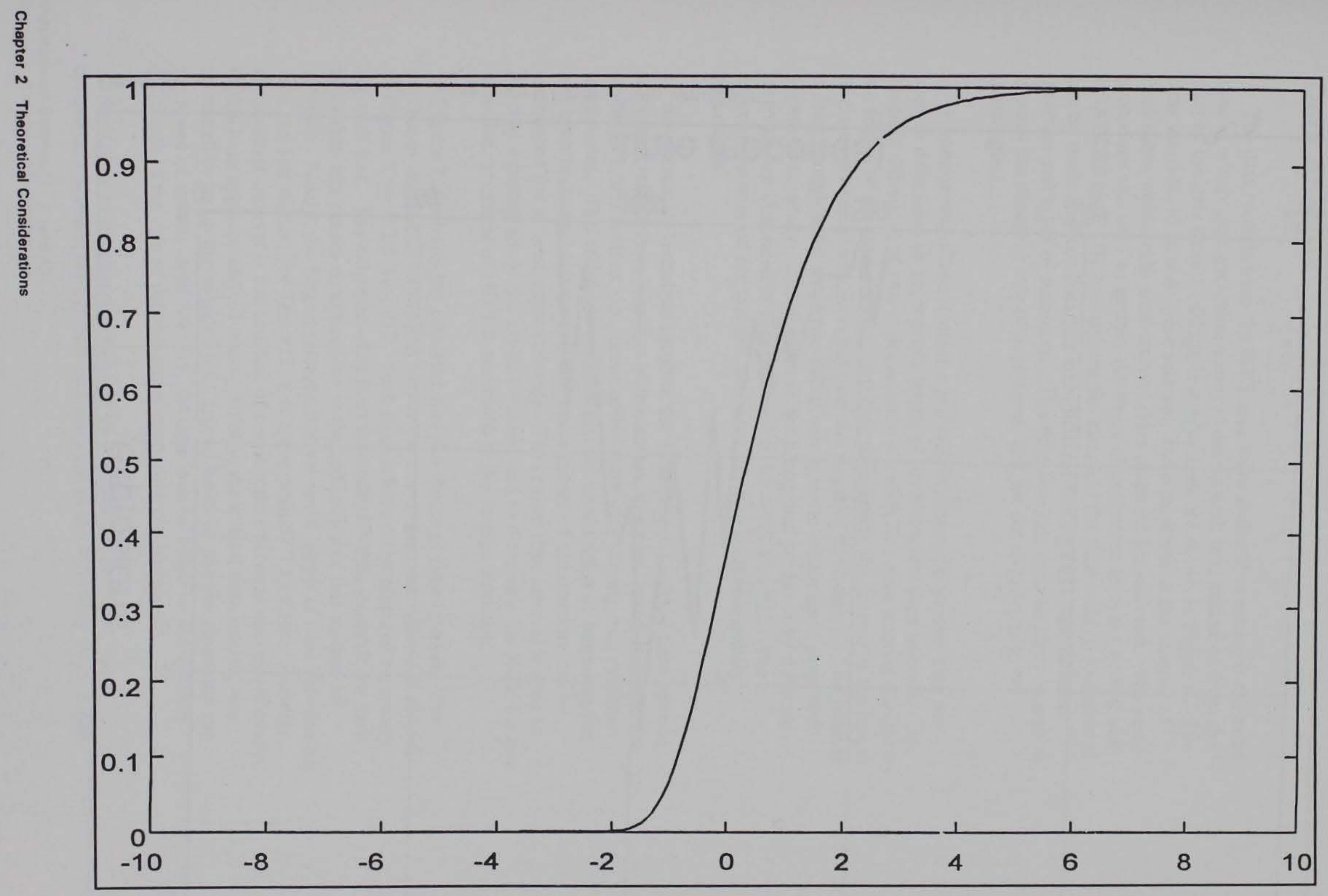

$\checkmark \quad$ Figure 2. The Gumbel Distribution $\exp (-\exp (-x))$ for a generic quantile $x$ 


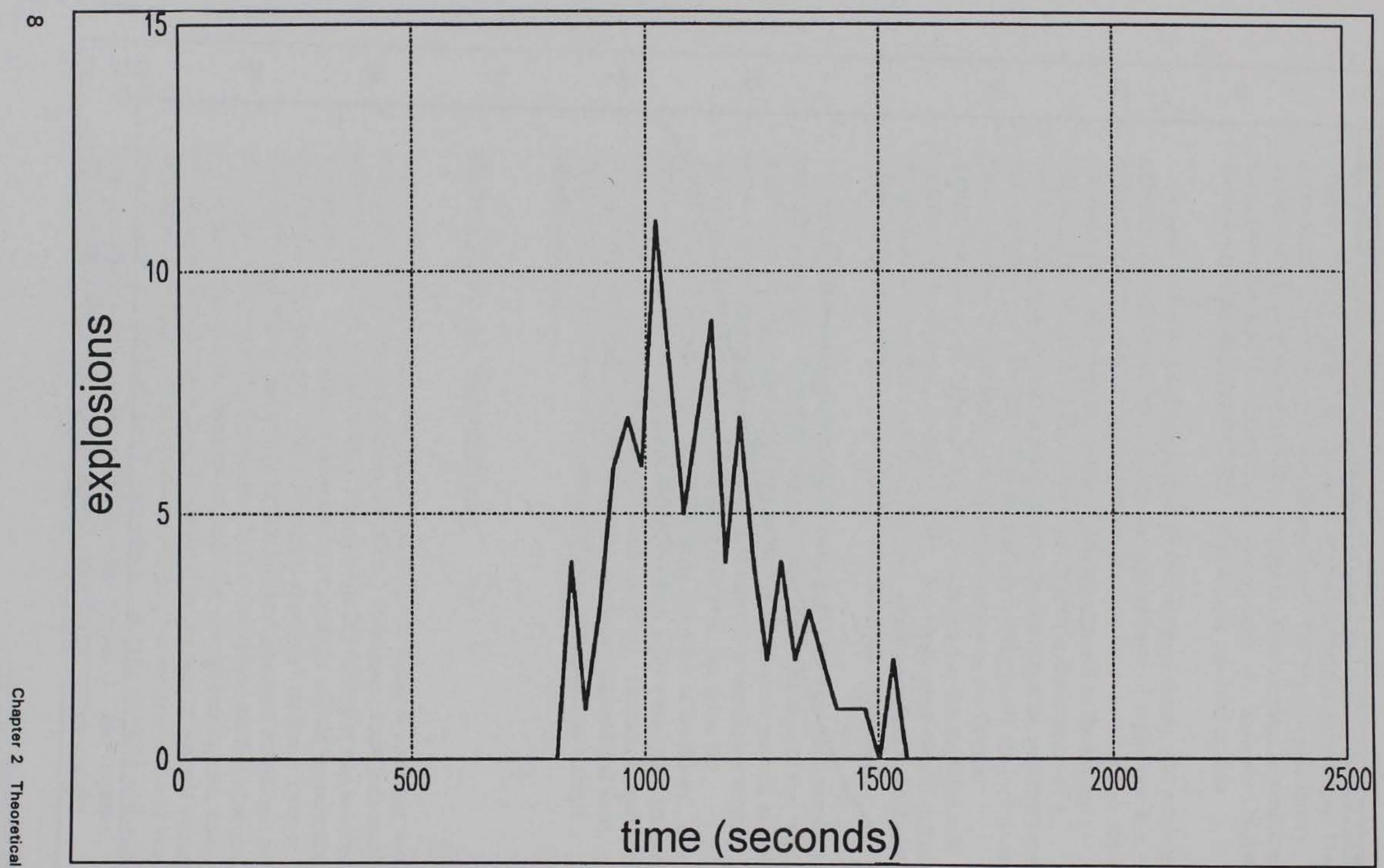

Figure 3. Histogram of explosion times from a Monte Carlo simulation 


\section{Data Analysis}

The data records from the WES tests were analyzed to establish the exact time at which each explosion occurred during each test, measured from the start of the data record. Example data records are shown in Figure 4. The time intervals of interest were selected, and histograms of the number of explosions within each time interval were prepared for each test. The same procedure was used to analyze the times of explosions from the NSWC and USATCES tests. By normalizing the rates and the time scale, a comparison can be made between data sets with different heating rates, and different numbers and types of munitions. The time intervals must be short enough to capture the changing rate of explosions, and yet not so short as to be meaningless.

A comparison of two distinct a priori definitions of explosion rate was used to determine an appropriate length of the histogram time interval. One possible definition of rate is the number of events in a time interval divided by the length of the time interval. Another definition is the inverse of the length of time between events averaged over the length of the interval. The interval is long enough when these two definitions agree to within some prescribed degree of accuracy. The changes in the histograms produced by different intervals are illustrated in Figures 5 and 6 for WES Test \#12. The appropriate interval for this test was determined to be approximately 10 seconds.

An alternative technique involves the definition of a critical time interval associated with failure response of the storage structure, based on properties of the structure such as size, construction materials, venting, and response frequencies. This technique assumes that the combination of fragmentation and blast pressure loadings that will cause failure of the structure can be established (or at least approximated). The critical time interval is used to develop a histogram of the explosion rates, and to determine the MCE for any specified quantities of HD 1.2 munitions in the storage structure.

Figure 7 illustrates the importance of the choice of time interval. The maximum number of explosions versus the size of the time interval is plotted in Figure 7 for WES Test \#12. Such figures have to be prepared separately for each test. The existence of at least four relevant time intervals for each individual test causes complications in the application of this method of analysis. Firstly, the largest relevant interval is the length of time between the first and last events; for Test \#12, it is approximately 7 minutes. Secondly, the smallest interval is the smallest discernible time between successive events; in this case approximately $7 \mathrm{msec}$. Thirdly, the critical time interval was estimated to be on the order of 0.01 second based on the container size and the speed of sound. And fourthly, the time interval based on the a priori definition of the rate of detonations is approximately 10 seconds.

Such plots of maximum events can be prepared for each test, but a comparison between tests is best accomplished in the following way. Since 


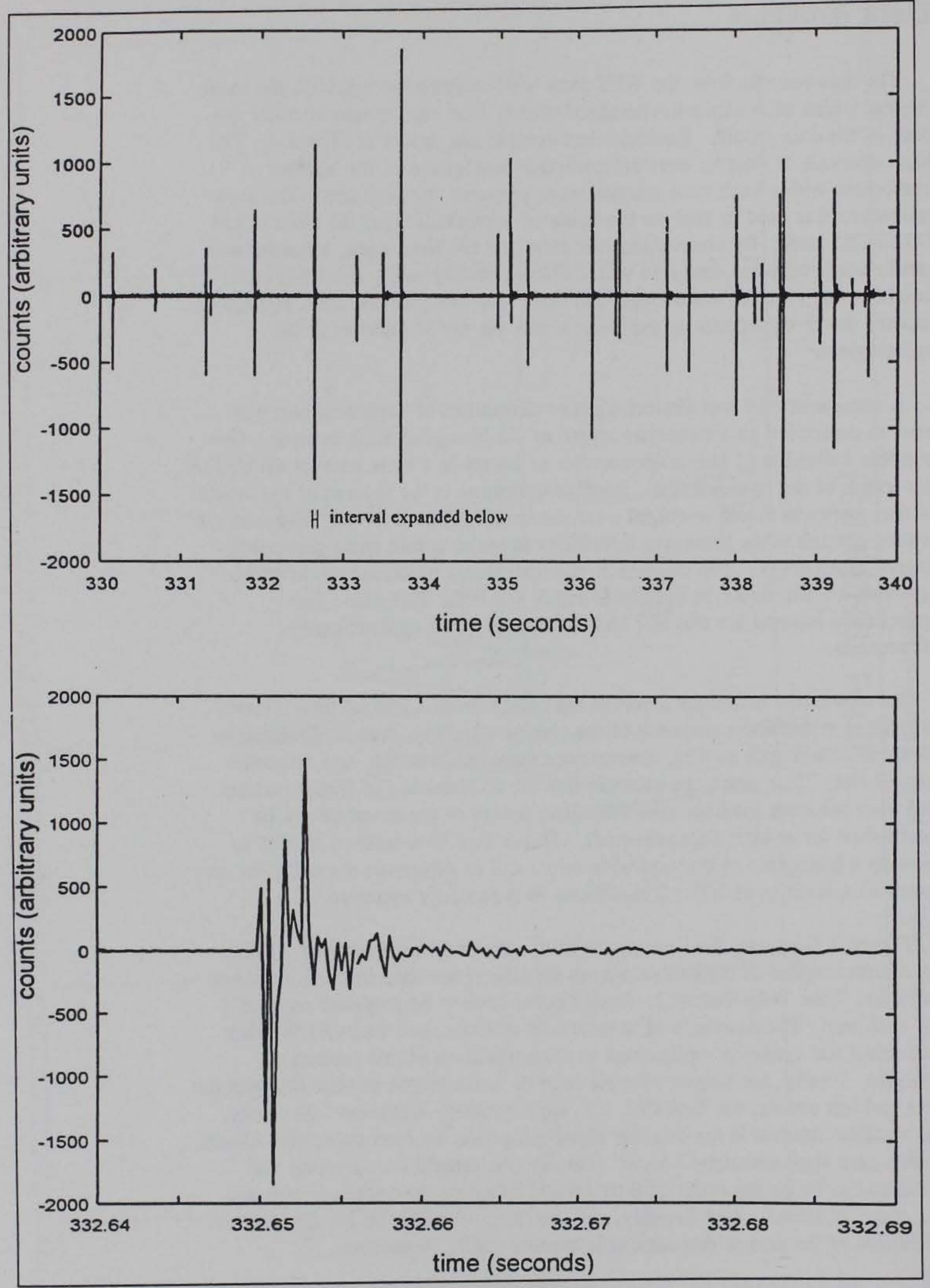

Figure 4. A portion of waveform from WES Test \#12 plotted at two scales 


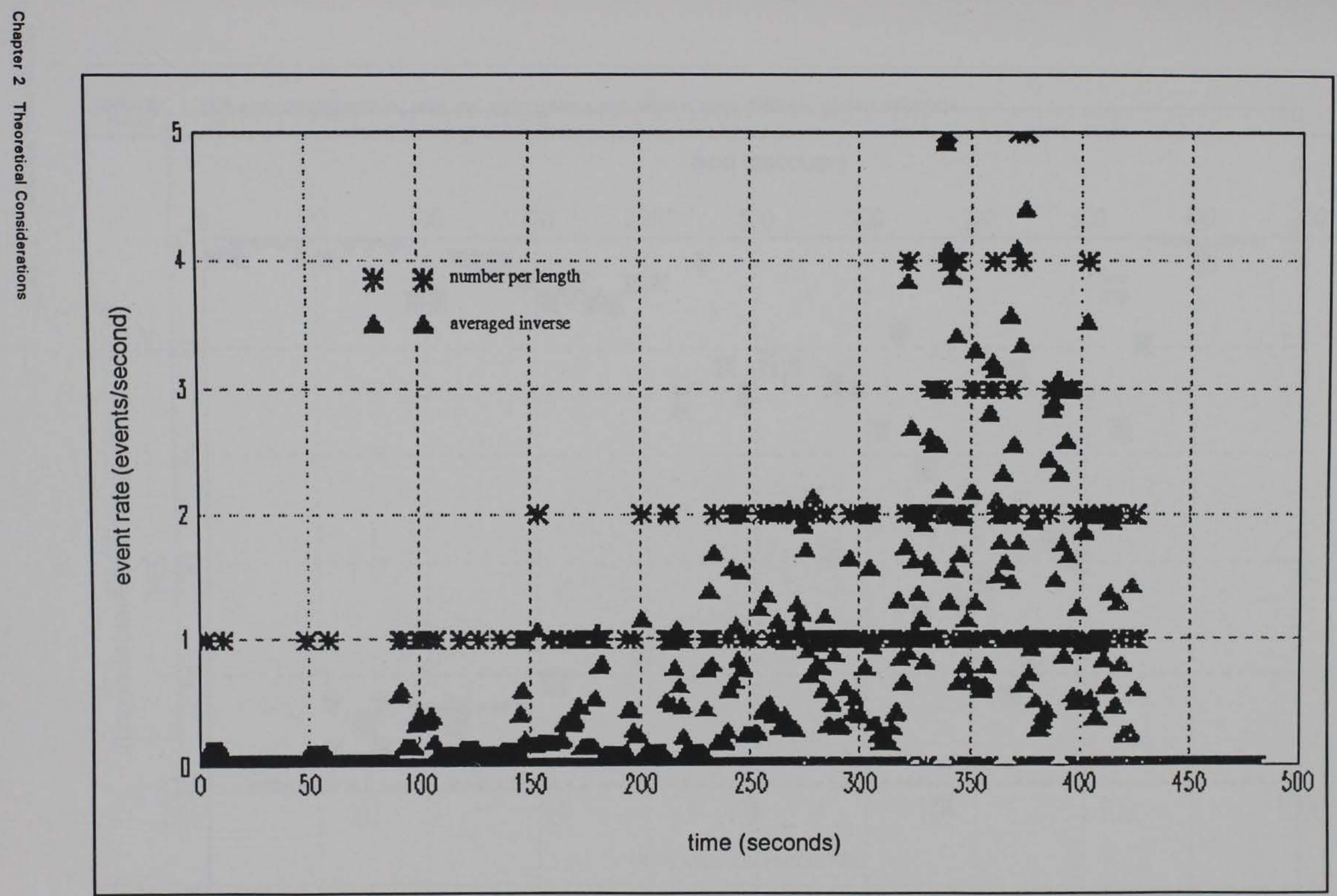

Figure 5. The two definitions of rate for WES Test \#12, with a time interval of one second 


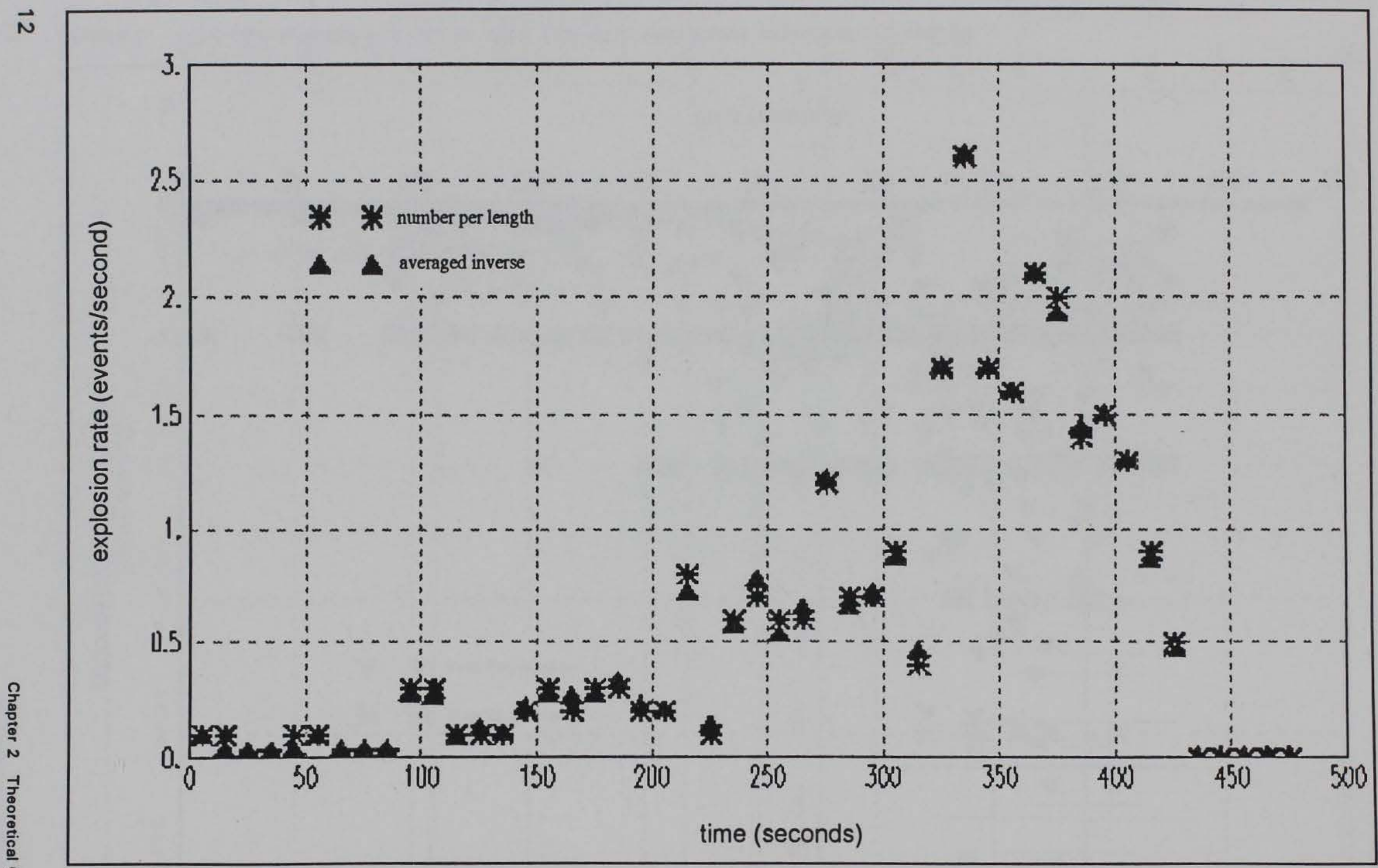

Figure 6. The two definitions of rate for WES Test \#12, with a time interval of ten seconds 


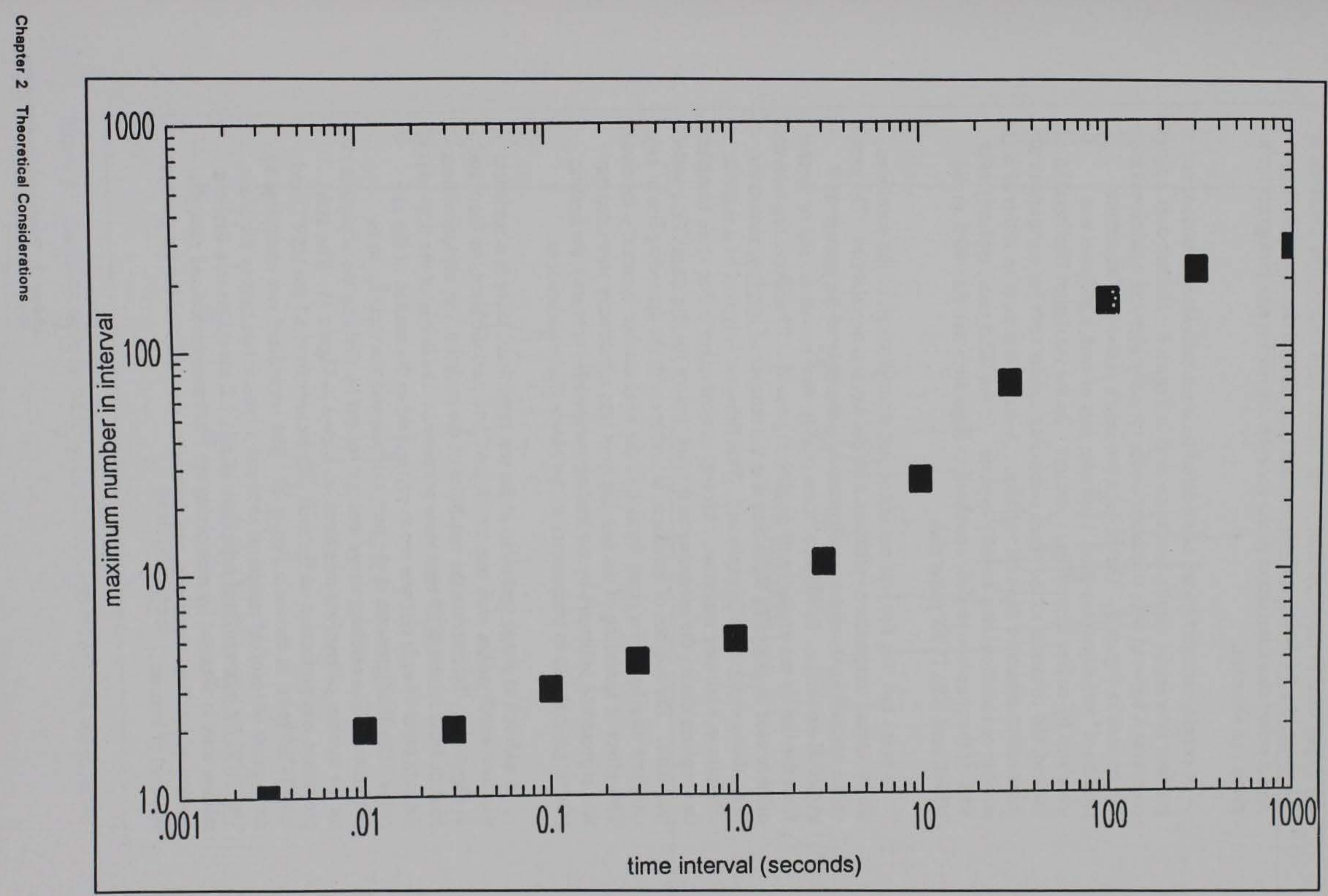


the values used in the calculation of the critical time interval are unknown at this point, the following analysis uses the time interval developed from the best $a$ priori characterization of the cook-off detonation rate histogram for a given fire situation.

A simple, straightforward comparison between individual tests does not produce meaningful results, as can be seen in Figure 8. Neither does a simple comparison between tests conducted under virtually identical conditions, as can be seen in Figure 9. Therefore, a procedure called the "maximum likelihood" technique was used. The time axis of each histogram was translated by adding appropriate constants. By the maximum likelihood method, the constants to use when combining similar tests for comparison are those which minimize the total variation. A factor that must be resolved is the number of munitions that actually explode. In the WES tests, typically over $80 \%$ of the rounds exploded, compared to (typically) less than $40 \%$ in the NSWC and USATCES pallet tests.

In every test, the fire was not distributed throughout all of the munitions, which caused temperature variations with geometrical correlations. The result of the nonuniform temperature distribution was a reduced percentage of exploded munitions. In addition, some rounds were moved around or ejected from the fire by the explosion of neighboring rounds. Therefore, the relevant number used in analyzing these data was the number of involved munitions (i.e., those which actually exploded). This behavior is typical of a realistic situation in a burning magazine. Another consideration is that some munitions can explode during the spreading of the fire, before the fire engulfs the entire magazine. The number of munitions in contact with the spreading fire at any one time may be only a small fraction of the total number eventually involved. This effect of spreading of the burning front was not directly simulated but was represented indirectly by the heating parameters--primarily the heating rate or general rise in temperature in the Monte Carlo simulations.

In addition to actual variations in the test conditions, there is a statistical variance which makes each test (or Monte Carlo run) different, as illustrated in Figure 10. To increase the reliability of the statistics, the explosion time records from all the WES tests were combined. The origin of the time axis of the rate plots of each test was set to correspond to the median of the rate curve. Small adjustments to the time axis allowed the variance to be minimized. The resulting curve was normalized by dividing the explosion rate by the number of involved munitions, as shown in Figure 11. The same procedure was performed on the total data records from all the NSWC and USATCES tests, as shown in Figure 12. The normalized rate multiplied by the number of involved munitions produces a typical rate history for a test. The MCE for heat-activated explosions of HD 1.2 munitions in a fire in a storage area is obtained by multiplying the maximum normalized rate, the number of involved munitions, and the critical time interval for the storage situation of interest. 

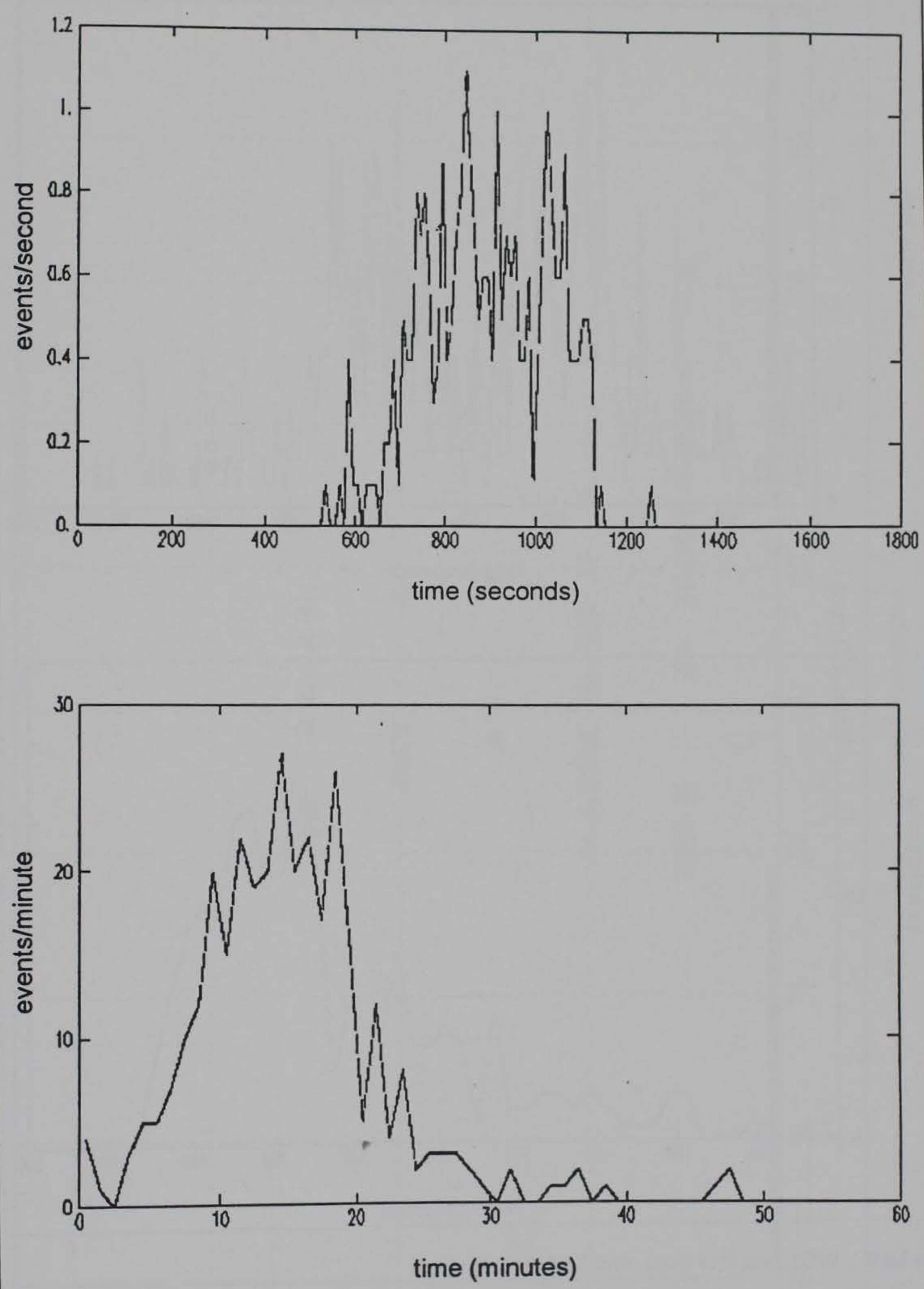

Figure 8. Explosion rates for WES Test \#13 (top), and NSWC Test \#6 (bottom) 


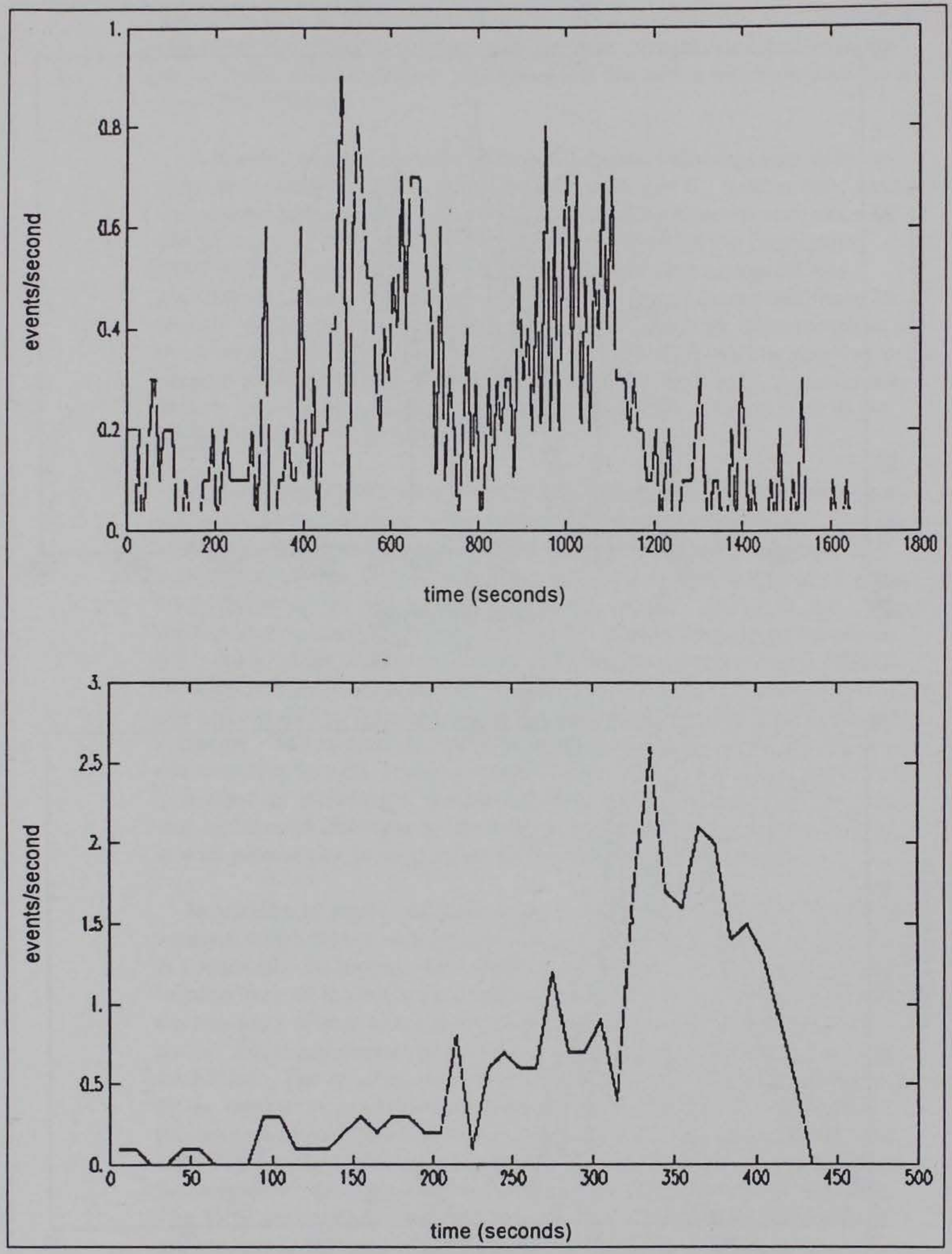

Figure 9. WES Test \#11 (top), and Test \#12 (bottom) 


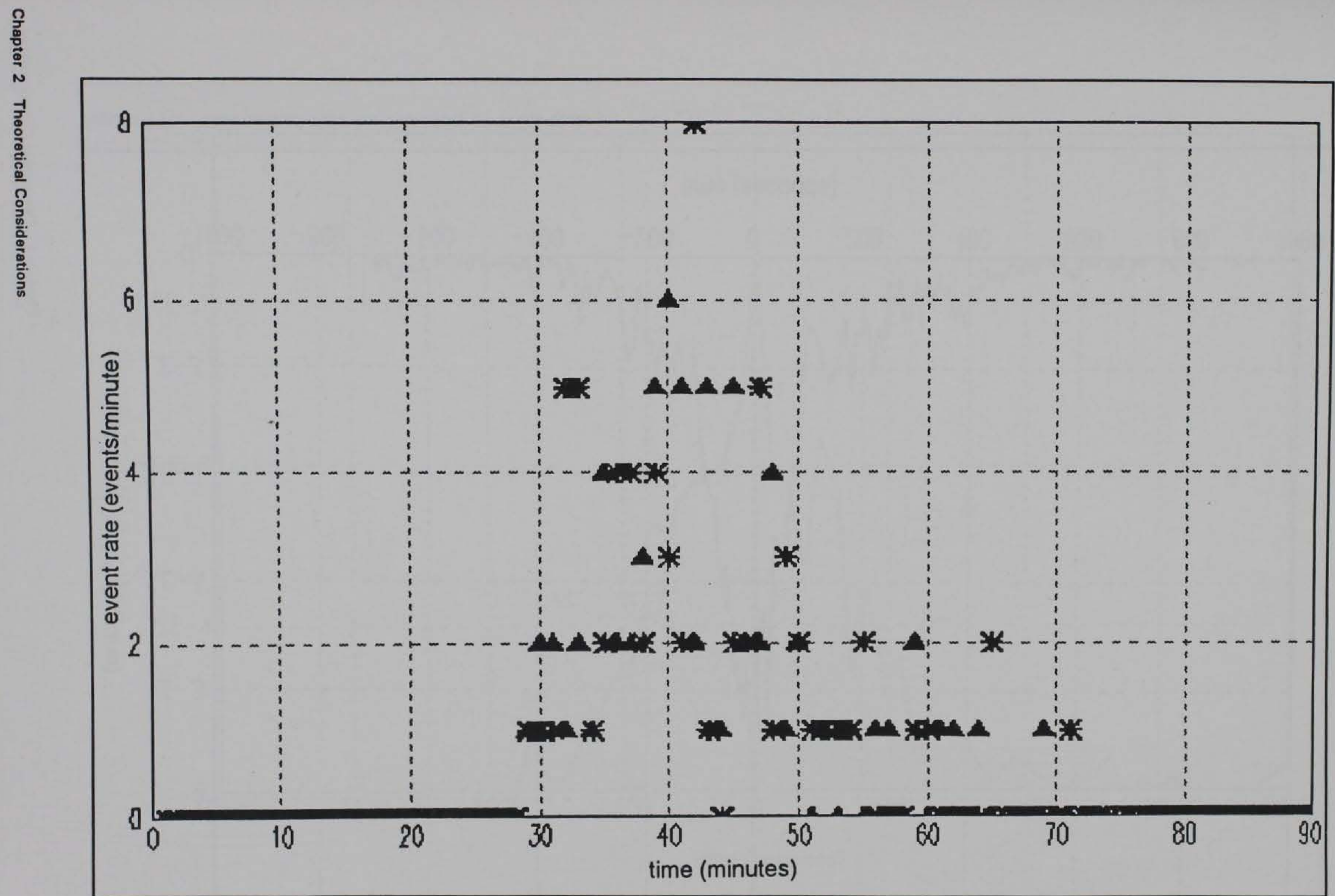

Figure 10. Two simulated tests of 70 munitions in identical fire conditions 


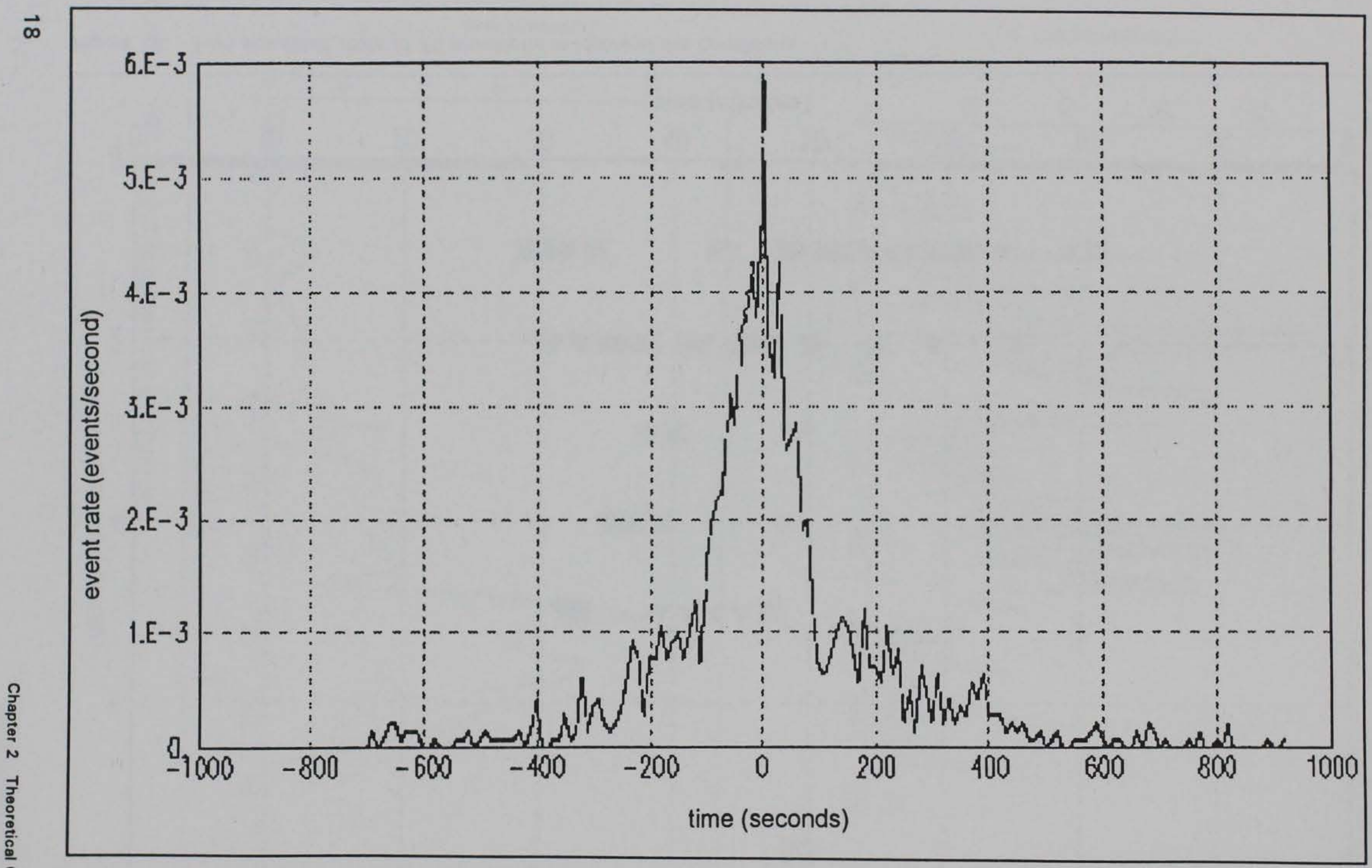

Figure 11. Normalized rate for the total of WES tests 


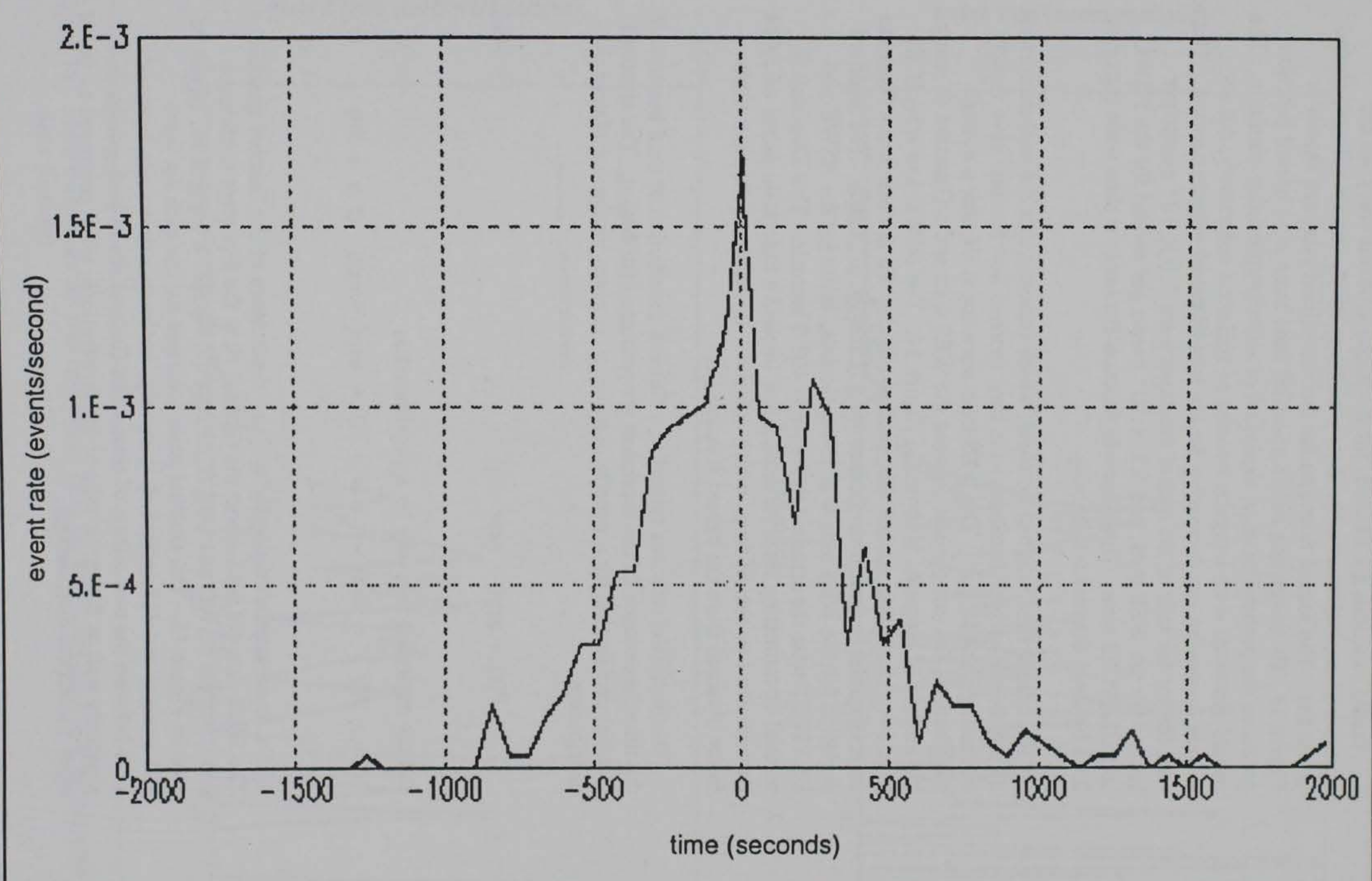


Hazard functions are defined, for the purpose of this study, as the normalized explosion rates for those munitions which remain yet unexploded in the fire. The hazard functions for the normalized rates are shown in Figure 13. By integrating over a specified time interval, a hazard function produces the probability of an explosion of any one remaining munition. The hazard functions were irregular because of statistical uncertainty, but are neither increasing nor decreasing for the tails of the distribution curves. The best fits for the tails of the hazard functions were $7.1 \times 10^{-3}$ events per second for the WES tests, and $3.5 \times 10^{-3}$ events per second for the NSWC and USATCES tests. These hazards function fits imply a time scale factor of 2.03 between the two sets of tests.

The hazard functions had no trend, which indicates that the explosion rates do not follow normal distributions in time (normal distributions have rapidly increasing hazard rates). The WES tests were not at all near a normal distribution. The comparison between the WES tests and a Gaussian fit using the method of moments is shown in Figure 14. The NSWC and USATCES tests were closer to a normal distribution, but also did not match the Gaussian fit at the usual 5-percent confidence level (Crow et. al.,1960). The standard deviation for the WES tests was 199.6 seconds, while for the NSWC and USATCES tests the standard deviation is 408.0 seconds. This Gaussian fit method of estimating the time scale factor implied a time scale factor of 2.044 between the two sets of tests, which compares favorably to the time scale factor obtained from the hazard function fits.

The explosion rate was related to a Gumbel distribution of time because of the time dependence of the maximum temperature distribution. The standard Gumbel distribution for a generic quantile $\mathrm{x}$ is given by the cumulative distribution

$$
F(x)=\exp (-\exp (-x))
$$

and the explosion rate may be approximated as

$$
\text { rate }=N_{0} / s * \exp (-(t / s+b)) * \exp (-\exp (-(t / s+b)))
$$

with a linear approximation for the time dependence of the Gumbel quantile. The WES data did not support the Gumbel fit at the 5-percent confidence level, but the NSWC and USATCES data did support a Gumbel fit, as can be seen in Figure 15. The Gumbel scale constants for the each set were 154 seconds and 317 seconds respectively, implying a time scale factor of 2.06 between the two groups of tests. The Gumbel fits to both groups do especially well at fitting the tails of the distributions, when compared to fits of other theoretical distributions. 


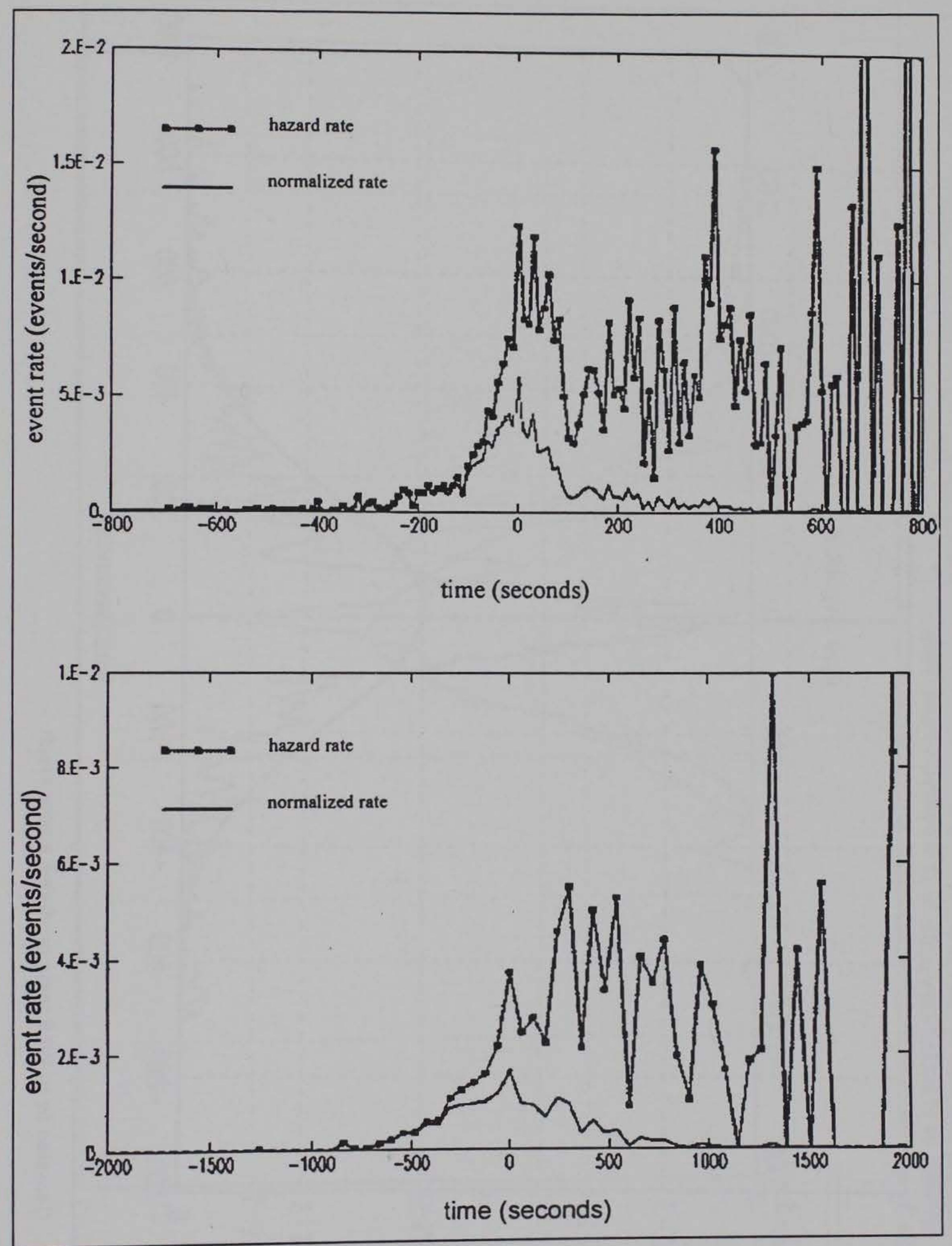

Figure 13. Hazard rates shown with normalized rates for WES tests (top) and NSWC-USATCES tests (bottom) 


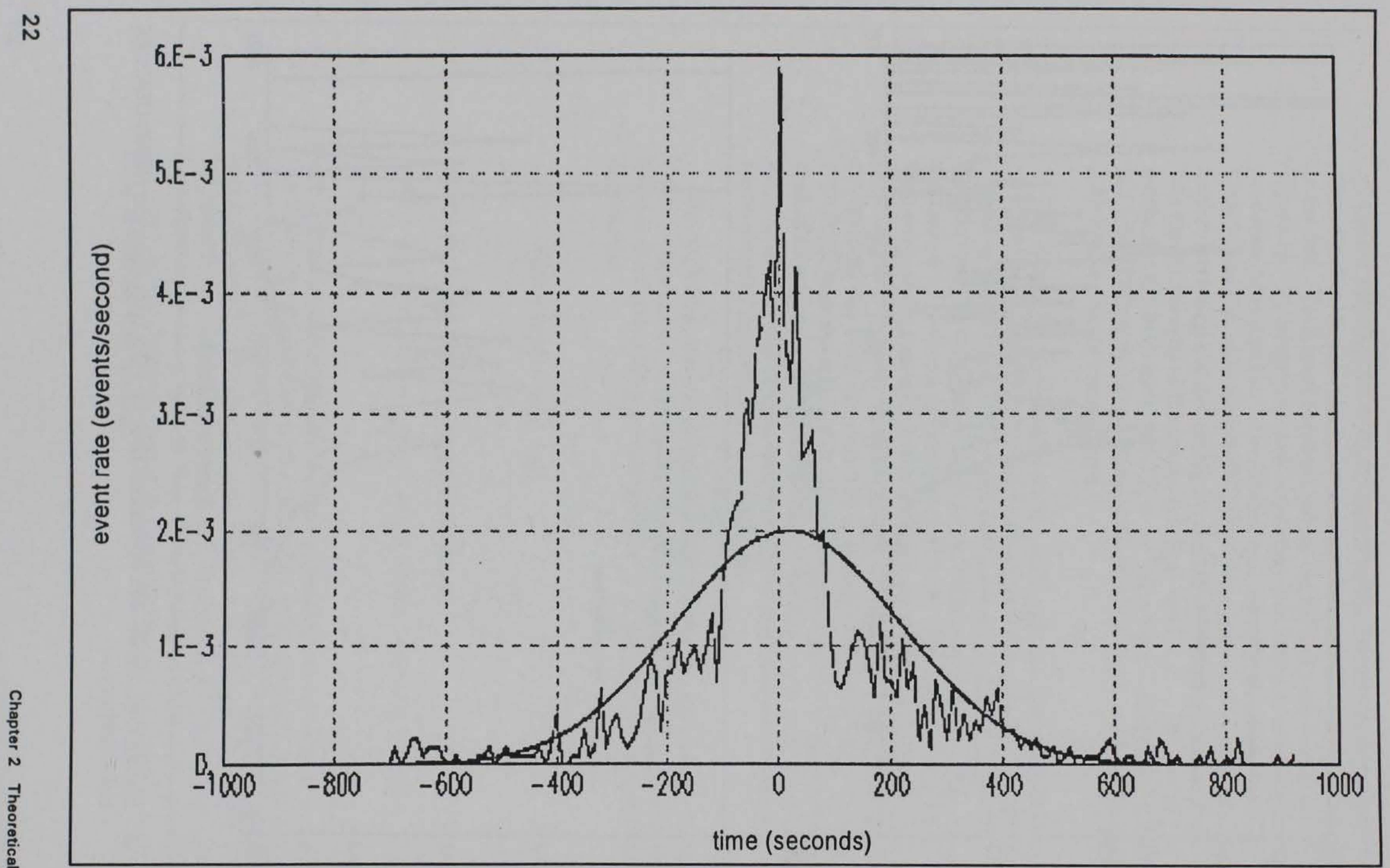

Figure 14. Gaussian fit to WES tests produced a poor result 


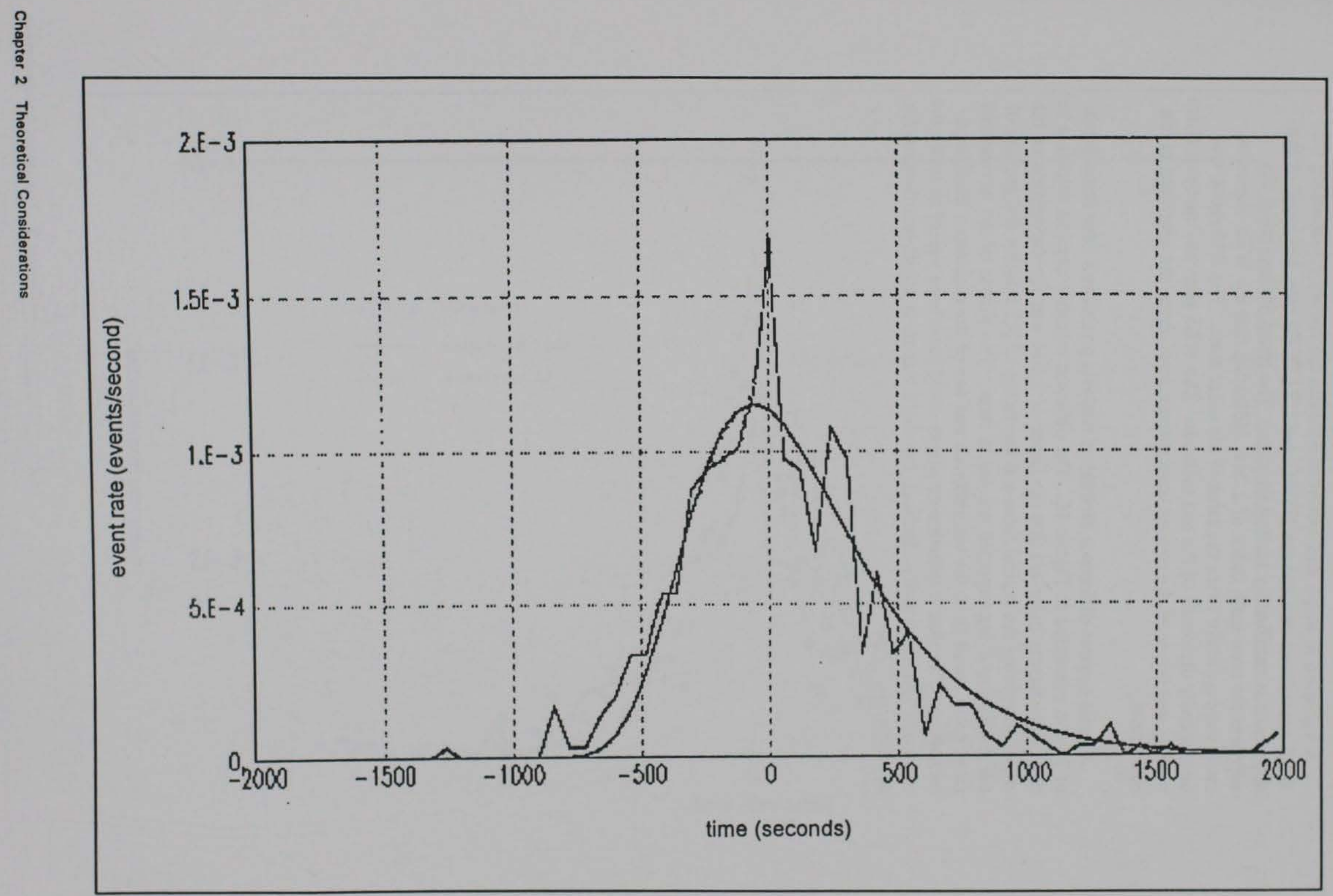

Figure 15. Gumbel fit to NSWC-USATCES tests produced a good result 
A variation in shape was exhibited between the curves representing the WES normalized rate and the NSWC and USATCES rate, but most of the difference is rectified by scaling the time. The trend of theoretical fits supports the time scale factor of 2.044, indicating that the WES explosion rates were typically twice the rates of the other tests. The difference was undoubtedly the result of the test methods. The WES tests had more uniform heating and fewer of the rounds were thrown away from the heat than in the other tests.

A least squares difference method of analysis produces a time scale factor of 3.1, as presented in Figure 16. The different methods used to calculate the time scale factors indicated that the factor of 2.044 was for the portion of the curves involving the typical rates and the factor of 3.1 was for the portion of the curves involving expected maximum rates. The shapes of the normalized rate curves result from the test methods and use of the maximum likelihood technique. The time translation constants could have been varied to make the two scale factors virtually identical, but there was no a priori justification for so doing. 


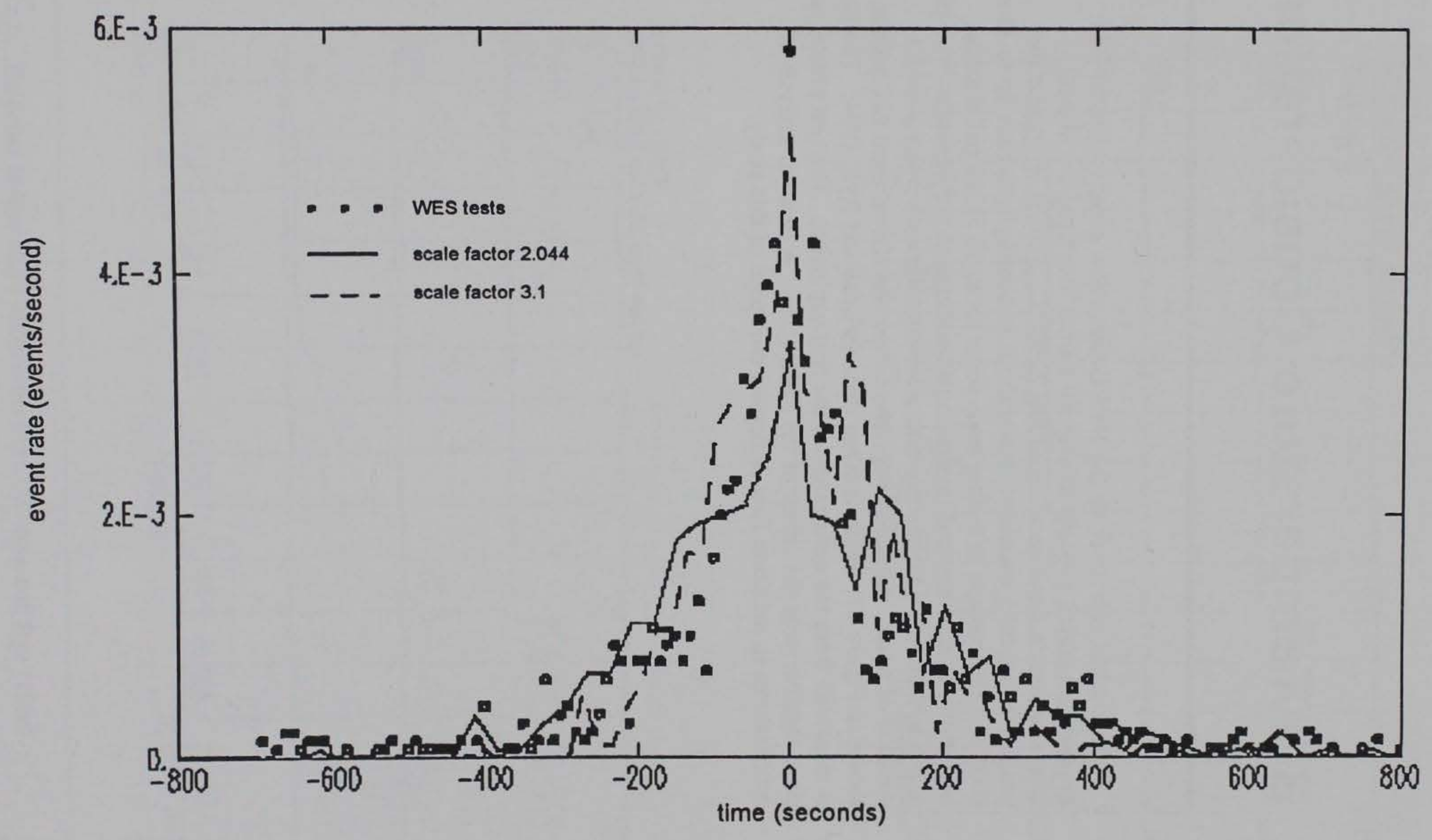

Figure 16. Comparison of WES tests and scaled NSWC-USATCES tests 


\section{Temperature Considerations}

The thermal records from the NSWC tests show great variability. In general, the probes exposed to external flames measured the highest temperatures, in some cases exceeding $1000{ }^{\circ} \mathrm{C}$. Probes in or near the projectiles typically measured temperatures increasing with time up to about $600{ }^{\circ} \mathrm{C}$. The method of testing made some locations in the pallet stacks cooler due to exposure and insulation. In addition to the variation from place to place, there were large temperature variations at each point over the duration of a test. Selected temperature-time waveforms from two probes are shown in Figure 17, which is taken from Swisdak and Rye, 1994. The time is measured from the occurrence of the first explosion. The two probes were close together near the center of the pallet stack. A detailed analysis of temperatures in munition fires is beyond the scope of this study. 


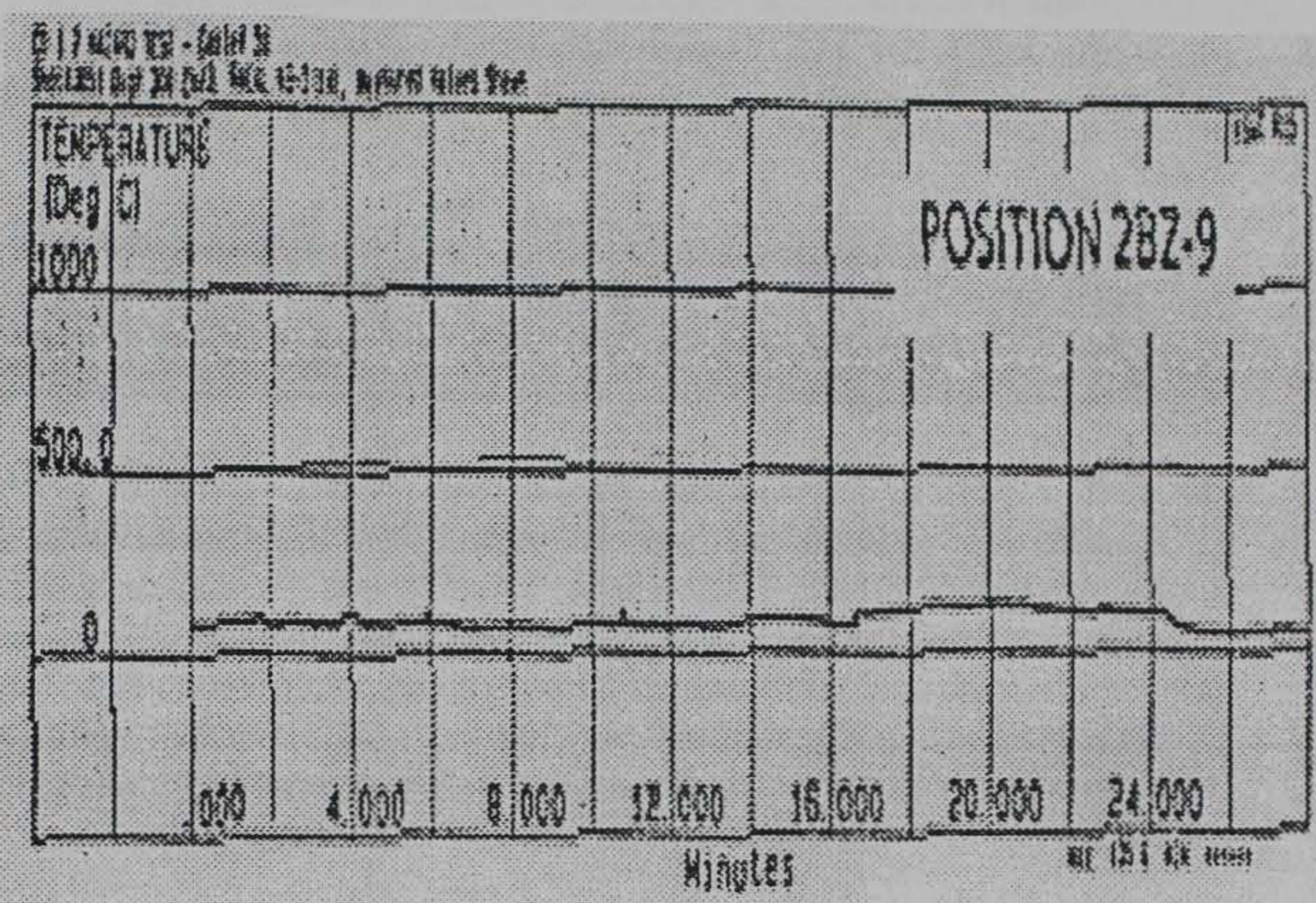

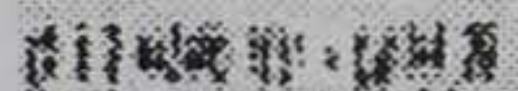

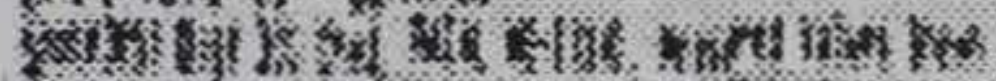

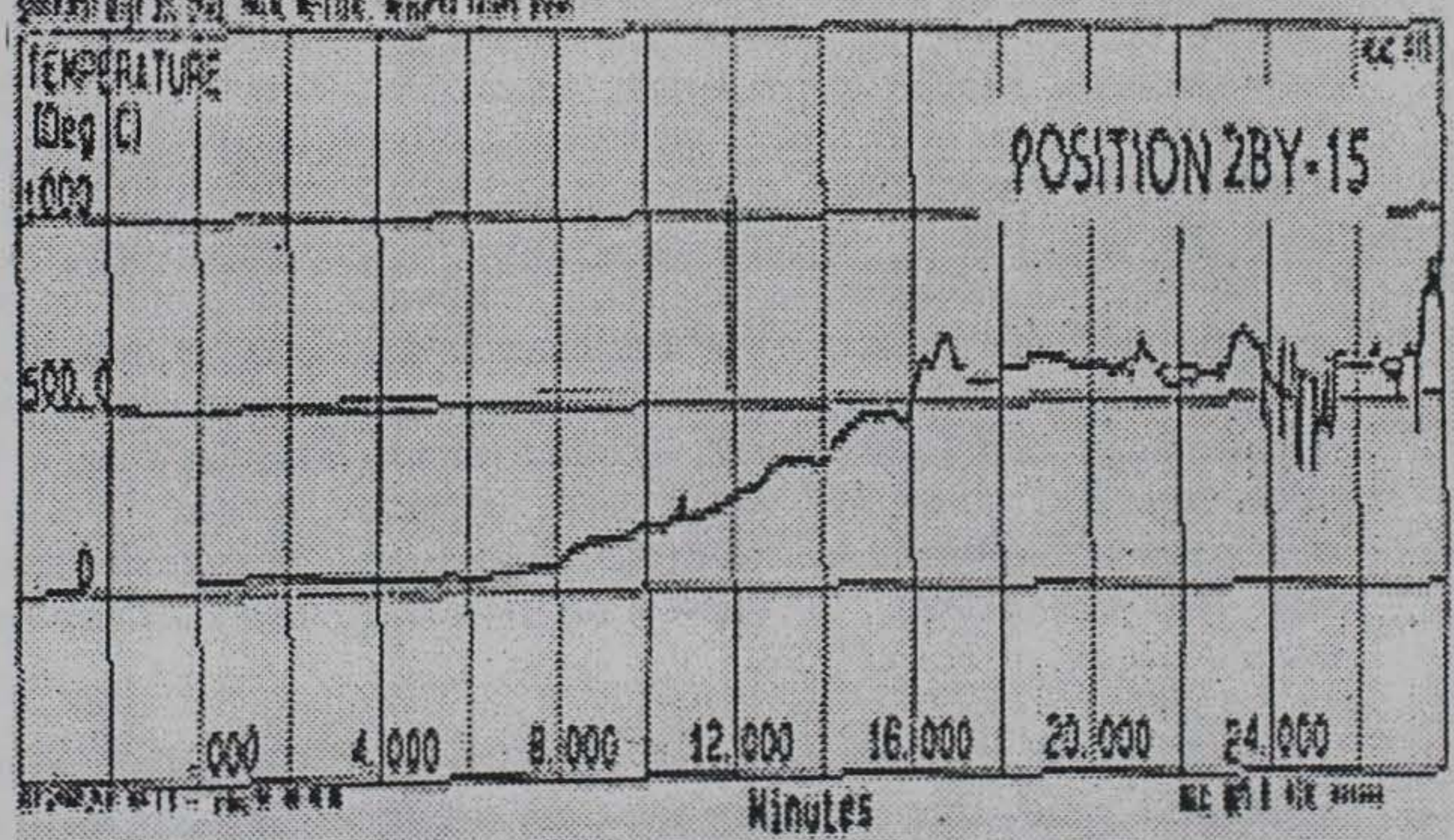

Figure 17. Selected temperature waveforms from Swisdak and Rye (1994) 


\section{Experimental Techniques}

A number of methods are commonly used for storing ammunition and explosives (DOD 6055.9-STD, 1992). Identifying the variation in hazards produced by the different storage conditions can be difficult. One technique that can be used to study such variation is by physically modeling different storage areas and configurations. Another method is to select typical or extreme conditions, and infer the results of differences through analysis of test data.

\section{NSWC and USATCES Tests}

The Palletized Load System is considered a typical method of storage by the Defense of Defense Explosive Safety Board (DDESB). The DDESB and the U.K. Explosive Storage and Transport Committee (ESTC) sponsored a series of tests of heat-activated explosions conducted in 1993-94 by the NSWC. The test program consisted of a series of open bonfire tests of palletized stacks of $105-\mathrm{mm}$ and 81-mm rounds (Swisdak and Rye, 1994). Similar bonfire tests of 120 -mm rounds were conducted in 1993 by the USATCES (Williams, 1994).

The major objectives of the pallet stack tests were the determination of explosive yield and the recovery of fragments, to determine the level of airblast and fragment hazards. Note that these munitions were tested with shipping plugs (as normally stored) without a primary initiating train, so most of the explosions did not have a high yield. A typical yield was approximately 10 percent, indicating that 10 percent of the explosive energy in each round was released. The times of the explosion events were used as the data for the rest of this analysis.

Small-arms ammunition rounds were tested at WES as a low-cost method to examine the statistics of rates of heat-activated explosion events in HD 1.2 munitions. Initial tests with live rounds in open fires indicated that the primary explosives (or primers) exploded first, so that heat-activated explosions in small arms ammunition, as usually stored, are high-yield. The explosions in the large projectiles tested by NSWC and USATECS probably would have been high-yield if their primers had been installed. Each 
explosion of a small-arms round typically produced three fragments. Tests with bare primers gave explosion results inconsistent with the live round tests, so the tests reported here were conducted with blank 0.223 and 0.308 caliber military rifle cartridges, for safety.

The ammunition rounds were oriented vertically with the primers down, and stacked in a single layer upon a 9-mm thick steel plate, which was placed $2 \mathrm{~cm}$ above the flames in a portable propane gas grill, with legs holding the grill $10 \mathrm{~cm}$ above the bare ground. After the fire was lit, the gas flow rate was set on high with the grill cover in place, and the starting time was marked. The rounds began to explode a few minutes later, and the sounds of the detonations were recorded by microphones located approximately $1 \mathrm{~m}$ from the grill, $3 \mathrm{~cm}$ above the ground, and pointed toward the grill. The signals were transmitted on coaxial cables into a 12-bit analog-to-digital board installed some $60 \mathrm{~m}$ away in a portable 386-based computer. Digitized samples were taken at $4096 \mathrm{~Hz}$ and stored on a hard disk. Temperatures inside the grill were measured with a multimeter, a thermocouple module, and $\mathrm{K}$ type probe. The measured temperatures varied depending on the placement of the probe, but were approximately $420{ }^{\circ} \mathrm{C}$ after 10 minutes of heating, when the tip of the probe was in contact with the steel plate.

After each test, the grill was allowed to cool and its contents were visually inspected. The cartridges suffered moderate damage from fragments of primers from other rounds. The effect of the vertical orientation of the rounds and direct contact of the primer with the metal plate was tested by placing the shells horizontally in several tests. No determinable effect on rates was noted. A total of 15 tests were conducted, and the number of rounds in a single test varied from 45 to 600 .

The digital recording began at the sound of the first explosion for each test. The resulting time history data were analyzed, and an elapsed time was determined for each succeeding explosion. The data were analyzed using an algorithm written to automatically identify the time of each explosion event. 


\section{Results of Data Analysis and Simulations}

The analysis of time histories of heat-activated explosions in HD 1.2 munitions led to a method of determining the MCE. The MCE was calculated by multiplying the maximum normalized rate of detonations, the critical time interval, and the number of munitions involved. A normalized rate can be obtained by statistical methods of data analysis for any given munitions storage condition.

The data obtained from the experiments described here were used to identify parameters such as heating rate, temperature variation, and maximum temperature, that are incorporated in the simulation program. Simulations of heat-activated explosions can predict explosion rates for a wide range of storage conditions not covered by experiments, and the validity of the results can be assessed by comparison to experimental data when available.

A large number of simulations were run with two sets of heat-activated explosion conditions similar to those in the tests. The problem of comparing individual simulation runs is the same as the problem encountered in comparing data from different tests. Histograms were calculated for the detonation rates of the simulated runs, which were processed with the same analysis routines as the field test data. The resulting normalized rates are comparable to the test results, as shown in Figure 18. The Gumbel-like curves produced by the simulations result in a closer fit to the NSWC and USATCES tests than to the WES tests, mainly because of the highly variable temperatures in the bonfire tests.

The plots in Figure 18 show up to a factor of two difference in peak value between the data and the simulations; however, this difference is not a flaw in the simulation program. The simulations were based on hypothetical input parameters which may not be the same as conditions under which tests were conducted. Therefore, since the general shapes of the data were simulated, the parameters were not adjusted further. If desired, the parameters could be refined to produce a better fit to any particular set of test data, but in general the simulation program should produce reasonable results. 


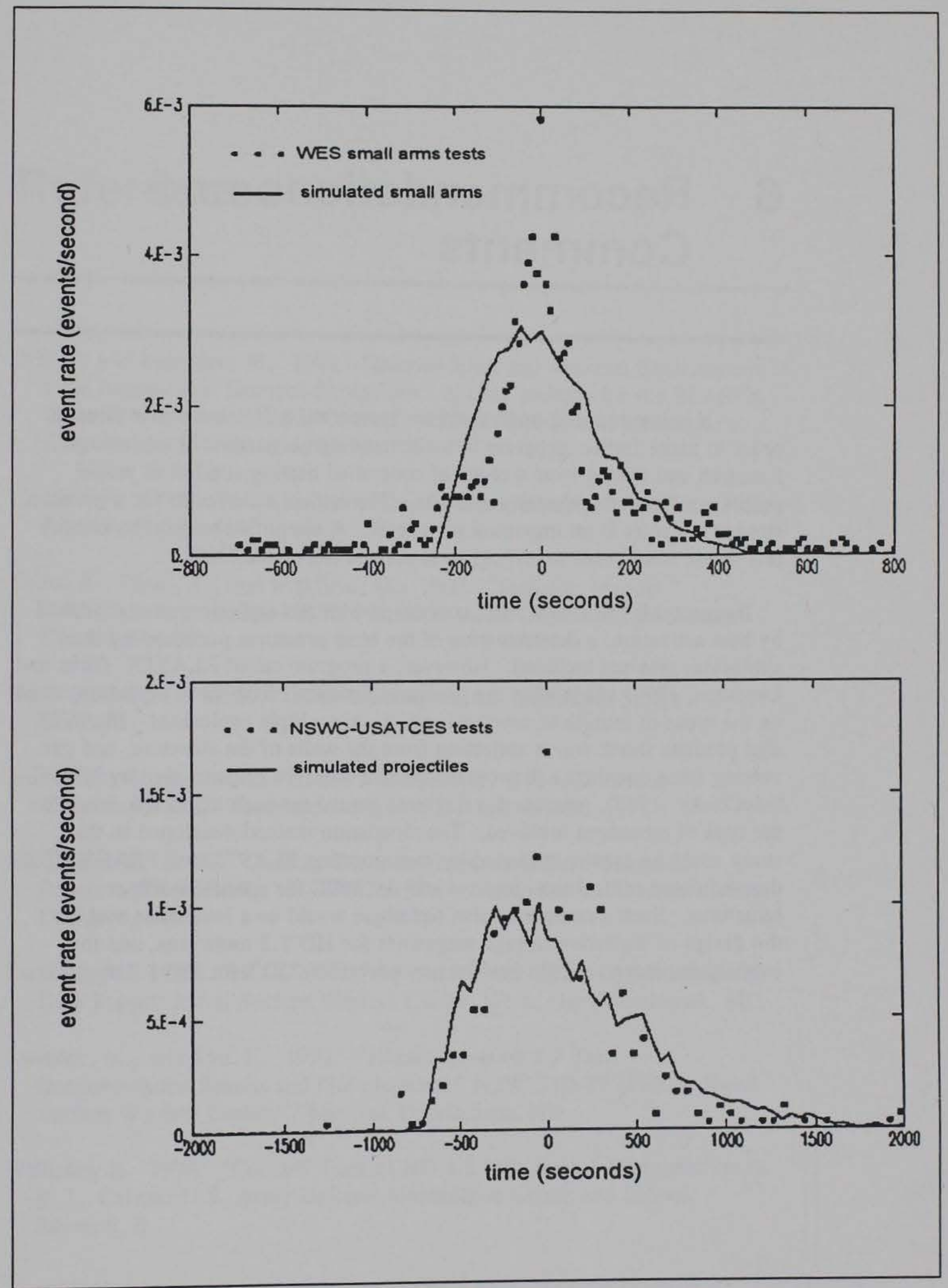

Figure 18. Comparison of actual and simulated tests 


\section{Recommendations and Comments}

It is important to understand the temperature distributions in fires in order to make further progress in understanding heat-activated explosions. Research and testing over a range of controlled heating conditions would enable a more comprehensive analysis. The critical time factor for a given storage structure is an important parameter. A simplified method to identify this factor would lead to an improved calculation of the MCE.

Because this effort was most concerned with the explosion rates produced by heat activation, a determination of the blast pressures produced by the explosions was not included. However, a program called BLASTX (Britt and Lumsden, 1994) can predict the pressures produced from each explosion based on the types of munitions involved, and from multiple explosions. BLASTX also predicts shock waves reflecting from the walls of the structure, and gas venting from openings. A program called FRAGHAZ, developed by NSWC (McClesky, 1988), predicts the fragment hazard for each explosion, based on the type of munitions involved. The simulation method developed in this study could be greatly enhanced by incorporating BLASTX and FRAGHAZ to determine the critical time interval and the MCE for specific storage structures. Such a comprehensive technique would be a invaluable tool for the design of explosive storage magazines for HD 1.2 munitions, and for establishing more realistic (and less conservative) QD's for HD 1.2 storage. 


\section{References}

Britt, J. and Lumsden, M. 1994. "Internal Blast and Thermal Environment from Internal and External Explosions: A User's Guide for the BLASTX Code, Version 3.0," SAIC 405-94-2; Science Applications International Corporation, St. Joseph, LA.

Castillo, E. 1988. "Extreme Value Theory in Engineering," Academic Press, Inc., San Diego, CA.

Crow, E., Davis, F., and Maxfield, M. 1960. "Statistics Manual," NAVORD Report 3369-NOTS 948; U.S. Naval Ordnance Test Station, China Lake, CA.

DOD 6055-9-STD 1992. "DOD Ammunition and Explosives Safety Standards."

Holtzclaw, H., Robinson, W., and Nebergall, W. 1984. "General Chemistry," D.C. Heath and Company, Lexington, MA.

McClesky, F. 1988. "Quantity-Distance Fragment Hazard Computer Program (FRAGHAZ)," NSWC TR 87-59; Naval Surface Warfare Center, Dahlgren, VA.

Swisdak, M. 1994. "HD 1.2 Test - 81 mm Mortar (Comp B)," Preliminary Data Report; Naval Surface Warfare Center, White Oak Detachment, MD.

Swisdak, M., and Rye, K. 1994. "Hazard Division 1.2 Tests Instrumentation Results and Interpretation," NSWCDD/TR-93/218; Naval Surface Warfare Center, White Oak Detachment, MD.

Williams, K. 1994. "Cookoff Tests of HD 1.2 Munitions," Memorandum to B. L. Carnes; U.S. Army Defense Ammunition Center and School, Savanna, IL. 

gathering and maintaining the data needed, and completing and reviewing the collection of information. Send comments regarding this burden estimate or any other aspect of thrs collection of intormation, including suggestions for reducing this burden. to Washington Headquarters services. Directorate for information Operations and Reports. 1215 jefferson Devis Highway, Suite 1204. Arlington, VA 22202-4302, and to the Office of Management and Budget, Paperwork Reduction Project (0704-0188), Washington, DC 20503.

\begin{tabular}{|l|c|c|}
\hline 1. AGENCY USE ONLY (Leave blank) & $\begin{array}{c}\text { 2. REPORT DATE } \\
\text { August } 1995\end{array}$ & $\begin{array}{c}\text { 3. REPORT TYPE AND DATES COVERED } \\
\text { Technical report }\end{array}$ \\
\hline
\end{tabular}

\section{TITLE AND SUBTITLE}

\section{FUNDING NUMBERS}

Analysis of Heat-Activated Explosions in Storage of HD 1.2 Munitions; SSES Report No. 1

6. AUTHOR(S)

Benny L. Carnes, John S. Furey, Morris P. Fields

7. PERFORMING ORGANIZATION NAME(S) AND ADDRESS(ES)

U.S. Army Engineer Waterways Experiment Station, 3909 Halls Ferry Road, Vicksburg, MS 39180-6199

Computer Science Corporation, Vicksburg, MS 39180-5378

Illinois Institute of Technology Research Institute,

Dayton, $\mathrm{OH} \quad 45432-3036$

\section{SPONSORING/MONITORING AGENCY NAME(S) AND ADDRESS(ES)}

Office of the Under Secretary of Defense for Acquisition,

Washington, DC 20315

U.S. Army Technical Center for Explosives Safety,

Savanna, IL 61074-9639
8. PERFORMING ORGANZATION REPORT NUMBER

Technical Report

SL-95-16

\section{SUPPLEMENTARY NOTES}

Available from the National Technical Information Service, 5285 Port Royal Road, Springfield, VA 22161.

\section{2a. DISTRIBUTION/AVAILABILITY STATEMENT}

12b. DISTRIBUTION CODE

Approved for public release; distribution is unlimited.

\section{ABSTRACT (Maximum 200 words)}

Several techniques for the analysis of heat-activated explosions of munitions are introduced to identify the significant parameters that control the resulting hazardous effects. These parameters are used in computer simulations to predict the rates of explosions of Hazard Division (HD) 1.2 munitions involved in storage facility fires. Data from bonfire tests of typical HD 1.2 munitions are analyzed and compared to "cook-off" tests of small arms rounds (also classed as HD 1.2). A comparison of measured and predicted explosion rates reveals that simulations can be used for reliable prediction of munition detonations. A computer simulation program, developed as part of this study, enables users to predict the explosion rates of HD 1.2 munitions in various quantities and combinations of storage conditions. Modules for prediction of explosion effects, such as airblast pressures/impulses and fragment hazards; can be incorporated to enhance the products of the calculations. As future tests are conducted, additional data can also be added to improve the statistical accuracy of the predictions.

\section{SUBNECT TERMS}

Accidental explosions

Ammunition fires
Explosion hazards

HD 1.2 munitions
15. NUMBER OF PAGES

40

16. PRICE CODE
17. SECURITY CLASSIFICATION OF REPORT
18. SECURITY CLASSIFICATION OF THIS PAGE

U1

